\title{
AGN and QSOs in the eROSITA All-Sky Survey
}

\section{Statistical properties}

\author{
Alexander Kolodzig ${ }^{1}$, Marat Gilfanov ${ }^{1,2}$, Rashid Sunyaev ${ }^{1,2}$, Sergey Sazonov ${ }^{2,3}$, and Marcella Brusa ${ }^{4,5,6}$ \\ ${ }^{1}$ Max-Planck-Institut für Astrophysik (MPA), Karl-Schwarzschild-Str. 1, 85741 Garching, Germany \\ e-mail: alex@mpa-garching.mpg.de \\ 2 Space Research Institute (IKI), Russian Academy of Sciences, Profsoyuznaya ul. 84/32, 117997 Moscow, Russia \\ 3 Moscow Institute of Physics and Technology, Institutsky per. 9, 141700 Dolgoprudny, Russia \\ 4 Max-Planck-Institut für extraterrestrische Physik (MPE), Giessenbachstrasse, Postfach 1312, 85741 Garching, Germany \\ 5 Dipartimento di Fisica e Astronomia, Universitá di Bologna, viale Berti Pichat 6/2, 40127 Bologna, Italy \\ ${ }^{6}$ INAF - Osservatorio Astronomico di Bologna, via Ranzani 1, 40127 Bologna, Italy
}

Received 10 December 2012 / Accepted 15 August 2013

\begin{abstract}
Context. The main element of the observing program of the Spectrum-Roentgen-Gamma orbital observatory is a four-year all-sky survey, in the course of which the entire sky will be scanned eight times.

Aims. We analyze the statistical properties of active galactic nuclei (AGN) and quasars/quasi-stellar objects (QSOs ) that are expected to be detected in the course of the eROSITA all-sky survey (eRASS).

Methods. According to the currently planned survey strategy and based on the parameters of the Galactic and extragalactic X-ray background as well as on the results of the recent calculations of the eROSITA instrumental background, we computed a sensitivity map of the eRASS. Using the best available redshift-dependent AGN X-ray luminosity function (XLF), we computed various characteristics of the eRASS AGN sample, such as their luminosity- and redshift distributions, and the brightness distributions of their optical counterparts.

Results. After four years of the survey, a sky-average sensitivity of about $1 \times 10^{-14} \mathrm{erg} \mathrm{s}^{-1} \mathrm{~cm}^{-2}$ will be achieved in the $0.5-2.0 \mathrm{keV}$ band. With this sensitivity, eROSITA is expected to detect about 3 million AGN on the extragalactic sky $\left(|b|>10^{\circ}\right)$. The median redshift of the eRASS AGN will be $z \approx 1$ with approximately $40 \%$ of the objects in the $z=1-2$ redshift range. About $10^{4}-10^{5}$ AGN are predicted beyond redshift $z=3$ and about 2000-30000 AGN beyond redshift $z=4$, the exact numbers depend on the poorly known behavior of the AGN XLF in the high-redshift and high-luminosity regimes. Of the detected AGN, the brightest $10 \%$ will be detected with more than $\approx 38$ counts per point-spread-function (half-energy width), while the faintest $10 \%$ will have fewer than $\approx 9$ counts. The optical counterparts of approximately $95 \%$ of the AGN will be brighter than $I_{\mathrm{AB}}=22.5 \mathrm{mag}$. The planned scanning strategy will allow one to search for transient events on a timescale of half a year and a few hours with a $0.5-2.0 \mathrm{keV}$ sensitivity of $\approx 2 \times 10^{-14}$ to $\approx 2 \times 10^{-13} \mathrm{erg} \mathrm{s}^{-1} \mathrm{~cm}^{-2}$, respectively.
\end{abstract}

Key words. surveys - X-rays: general - X-rays: galaxies - quasars: general - galaxies: luminosity function, mass function galaxies: active

\section{Introduction}

Large samples of X-ray detected active galactic nuclei (AGN) combined with follow-up optical data for identifying objects and their redshift determination are fundamental for understanding AGN evolution and the growth of supermassive black holes with cosmic time. These samples are constructed in various extragalactic X-ray surveys spanning from wide-shallow to narrowdeep surveys. Many of these have been conducted in the past decade with the Chandra and XMM-Newton X-ray observatories, which were instrumental in understanding the cosmic X-ray background and evolution of AGN at low- and high redhifts (Brandt \& Hasinger 2005). While Chandra and XMM-Newton have now surveyed several hundreds of square degrees (e.g. $\sim 360 \mathrm{deg}^{2}$ of the 2XMM-catalog, Watson et al. 2009), the sensitivity of the archival observations is far from homogeneous. Moreover, the sky area covered by largest contiguous surveys did not exceed several (e.g. XMM-COSMOS: Hasinger et al. 2007; XBootes: Murray et al. 2005) to several tens of square degrees (e.g. XMM-LSS: Chiappetti et al. 2012; XWAS: Esquej et al. 2013; XXL: Pierre et al. 2011), which in particular limited our knowledge of the evolution and clustering properties of the most luminous AGN severely. The most recent all-sky survey (Voges et al. 1999) in the X-ray band was performed by ROSAT $^{1}$ (Truemper 1993) more than two decades ago, creating an increasing demand for an all-sky survey to be conducted by the new generation of X-ray telescopes.

The eROSITA ${ }^{2}$ telescope (extended ROentgen Survey with an Imaging Telescope Array) will be able to statisfy this demand. It is the main instrument aboard the Russian SpektrumRoentgen-Gamma satellite ${ }^{3}$, which is scheduled for launch in 2014. Its main science goals are cosmological studies of clusters of galaxies and AGN, with the aim of constraining the nature of dark matter and dark energy. To achieve these goals,

\footnotetext{
http://www2011.mpe.mpg.de/xray/wave/rosat/

2 http://www.mpe.mpg.de/eROSITA

http://hea.iki.rssi.ru/SRG
} 
eROSITA will perform an all-sky survey (eRASS) during the first four years of its operation, followed by a phase of pointed observations. The main mission parameters and the telescope are described in the eROSITA Science Book (Merloni et al. 2012, hereafter SB).

In this study we explore the main statistical properties of the AGN sample that is expected to be detected in the course of the eRASS, including its luminosity- and redshift distributions. This will help to understand the capabilities of the eROSITA mission and, potentially, to tune the survey strategy and its parameters.

We use the following cosmological parameters throughout: $H_{0}=70.0 \mathrm{~km} \mathrm{~s}^{-1} \mathrm{Mpc}^{-1}, \Omega_{m}=0.30, \Omega_{\Lambda}=0.70$, $\Omega_{k}=0$. These values are commonly used for XLF modeling of AGN. We use the decimal logarithm throughout. The calculations are performed for two energy bands - soft $(0.5-2.0 \mathrm{keV})$ and hard $(2.0-10.0 \mathrm{keV})$. In computing count rates we used the most recent response matrix of eROSITA, erosita_iv_7telfov_ff.rsp ${ }^{4}$. As is appropriate for the allsky survey data analysis, this response matrix is averaged over the field-of-view and scaled to the seven eROSITA telescopes. In this work, we assume that the data from the entire survey of eROSITA is available for analysis.

\section{Sensitivity}

The point-source detection sensitivity of eROSITA in the allsky survey was discussed in detail by Prokopenko \& Gilfanov (2009). Since then, the spacecraft orbit has been changed to the L2 orbit and detailed calculations of the instrumental background became available. We therefore update the calculations of Prokopenko \& Gilfanov (2009) below. We first compute the sky average values (Sects. 2.1-2.3) and then calculate a more realistic sensitivity map (Sect. 2.4), which is used for our eRASS AGN calculations.

\subsection{Instrumental and cosmic background}

The eROSITA background is dominated by the photon background below $\sim 2 \mathrm{keV}$ and by the particle component above this energy. In the subsequent calculations we used the field-of-viewaveraged angular resolution $\left(\mathrm{PSF}^{5} \mathrm{HEW}^{6}\right.$ ) of $30^{\prime \prime}$ diameter for the soft band and 40" diameter in the hard band (Friedrich et al., priv. comm.). The corresponding PSF HEW areas are $\approx 707$ and $\approx 1257 \mathrm{arcsec}^{2}$, respectively. For the eRosita focal length, $1 \mathrm{~mm}$ on the detector corresponds to $\approx 128.8$ arcsec.

The instrumental non X-ray (particle) background spectrum is nearly flat in the counts space, with a normalization of $\approx 6.1 \times 10^{-3} \mathrm{cts} \mathrm{s}^{-1} \mathrm{~cm}^{-2} \mathrm{keV}^{-1}$ (Perinati et al. 2012). Within the PSF HEW it produces count rates of $\approx 2.7 \times 10^{-5}$ and $\approx 2.6 \times 10^{-4} \mathrm{cts} \mathrm{s}^{-1}$ for the soft and hard band for seven telescopes. Solar-activity-induced background events are not taken into account in this calculation, they are roughly accounted for via the observing efficiency introduced in the survey exposure time calculations. These numbers are the result of purely theoretical calculations and there are no direct measurements of the real background of an X-ray detector in the L2 orbit. Therefore the above numbers may have to be revised after the eROSITA launch.

The X-ray photon background (CXB) has two components (Lumb et al. 2002): (1) the truly diffuse Galactic background of

\footnotetext{
4 http://www2011.mpe.mpg.de/erosita/response/

5 PSF - Point spread function.

6 HEW - Half-energy width, alias half-power diameter (HPD).
}

local ionized interstellar medium (ISM) with a soft thermal spectrum, and (2) the hard power-law extragalactic CXB component.

To estimate the contribution of the ionized ISM emission we used the spectral fits from Lumb et al. (2002, Table 3) and obtained a count rate of $\approx 1.8 \times 10^{-4} \mathrm{cts} \mathrm{s}^{-1}$ within the PSF HEW for the soft band, assuming the same Galactic absorption $\left(N_{\mathrm{H}}=1.7 \times 10^{20} \mathrm{~cm}^{-2}\right)$ and solar abundance (Anders \& Grevesse 1989) as Lumb et al. (2002). Its contribution to the hard band can be neglected. As a caveat we note that the Lumb et al. (2002) analysis was based on the XMM-Newton observations of several blank fields located at high Galactic latitudes. Therefore these numbers should be considered as approximate, because they do not account for the inhomogeneity of the Galactic background radiation.

For the extragalactic $\mathrm{CXB}$ component we assumed a powerlaw spectrum with a photon index of $\Gamma=1.42$ (Lumb et al. 2002, Table 3). The power-law was normalized to the extragalactic CXB flux of $7.53 \times 10^{-12}$ and $2.02 \times 10^{-11} \mathrm{erg} \mathrm{s}^{-1} \mathrm{~cm}^{-2} \mathrm{deg}^{-2}$ for the soft and hard band (Moretti et al. 2003) ${ }^{7}$. We furthermore assumed Galactic absorption of $N_{\mathrm{H}}=6 \times 10^{20} \mathrm{~cm}^{-2}$ corresponding to the arithmetic mean of the $N_{\mathrm{H}}$-map of Kalberla et al. (2005) for the extragalactic sky $\left(|b|>10^{\circ}\right)$. With these parameters, the average count rate of extragalactic CXB within the PSF HEW (seven telescopes) is $\approx 2.8 \times 10^{-4}$ and $\approx 5.8 \times 10^{-5} \mathrm{cts} \mathrm{s}^{-1}$ in the soft and hard band, respectively.

In computing the contribution of extragalactic sources, one needs to take into account that a fraction of the background AGN will be resolved by eROSITA. Therefore, these sources will not contribute to the unresolved image background, which will affectg the point-source detection sensitivity. This effect reduces the extragalactic background count rate. At the average four year survey sensitivity of eROSITA (see Sect. 2.2) the resolved extragalactic CXB fraction ${ }^{8}$ achieves $\approx 31 \%$ in the soft and $\approx 6 \%$ in the hard band. The fractions were calculated using the number counts of Georgakakis et al. (2008, hereafter G08) and the extragalactic CXB flux of Moretti et al. (2003). Thus, the final values of the average count rate of the unresolved $\mathrm{CXB}$ emission within the PSF HEW is $\approx 1.9 \times 10^{-4}$ and $\approx 5.4 \times 10^{-5} \mathrm{cts} \mathrm{s}^{-1}$ in the soft and hard band, respectively.

To examine the background model, we used ROSAT allsky maps of diffuse X-ray emission (Snowden et al. 1997). We combined PSPC maps ${ }^{9}$ in the energy bands from $R 4$ to $R 7$ (see Snowden et al. 1997, Table 1) to cover the 0.44-2.04 keV range, which presents a reasonable approximation of the eROSITA soft band. The median count rate on the combined map is $\approx 0.87$ PSPC cts s$^{-1} \mathrm{deg}^{-2}$ for the extragalactic sky $(|b|>$ $\left.10^{\circ}\right)$. A convolution of our X-ray background model with the ROSAT PSPC response matrix (pspcb_gain2_256.rsp ${ }^{10}$ ) gives a $0.44-2.04 \mathrm{keV}$ count rate of $\approx 0.99 \mathrm{cts} \mathrm{s}^{-1} \mathrm{deg}^{-2}$, which is sufficiently close to the median value in the ROSAT map. ROSAT maps show moderate brightness variations in which $90 \%$ of the extragalactic sky count rate are between $\approx 0.34$ and

\footnotetext{
7 Strictly speaking, these flux values correspond to a slightly steeper slope $(\approx 1.45)$ of the CXB spectrum than the conventional value of 1.42 . This discrepancy reflects the uncertainty in the absolute CXB flux determinations. We nevertheless used them for consistency with the resolved fraction calculations below.

8 Note that Fig. 5 in Moretti et al. (2003) gives resolved fractions of $\sim 50 \%$ and $\sim 10 \%$, respectively. This difference also reflects the uncertainties of the CXB measurements.

9 http://www . xray.mpe.mpg.de/rosat/survey/sxrb/12/ass. html

${ }^{10} \mathrm{ftp}: / /$ legacy.gsfc.nasa.gov/caldb/data/rosat/pspc/ $\mathrm{cpf} /$ matrices/
} 


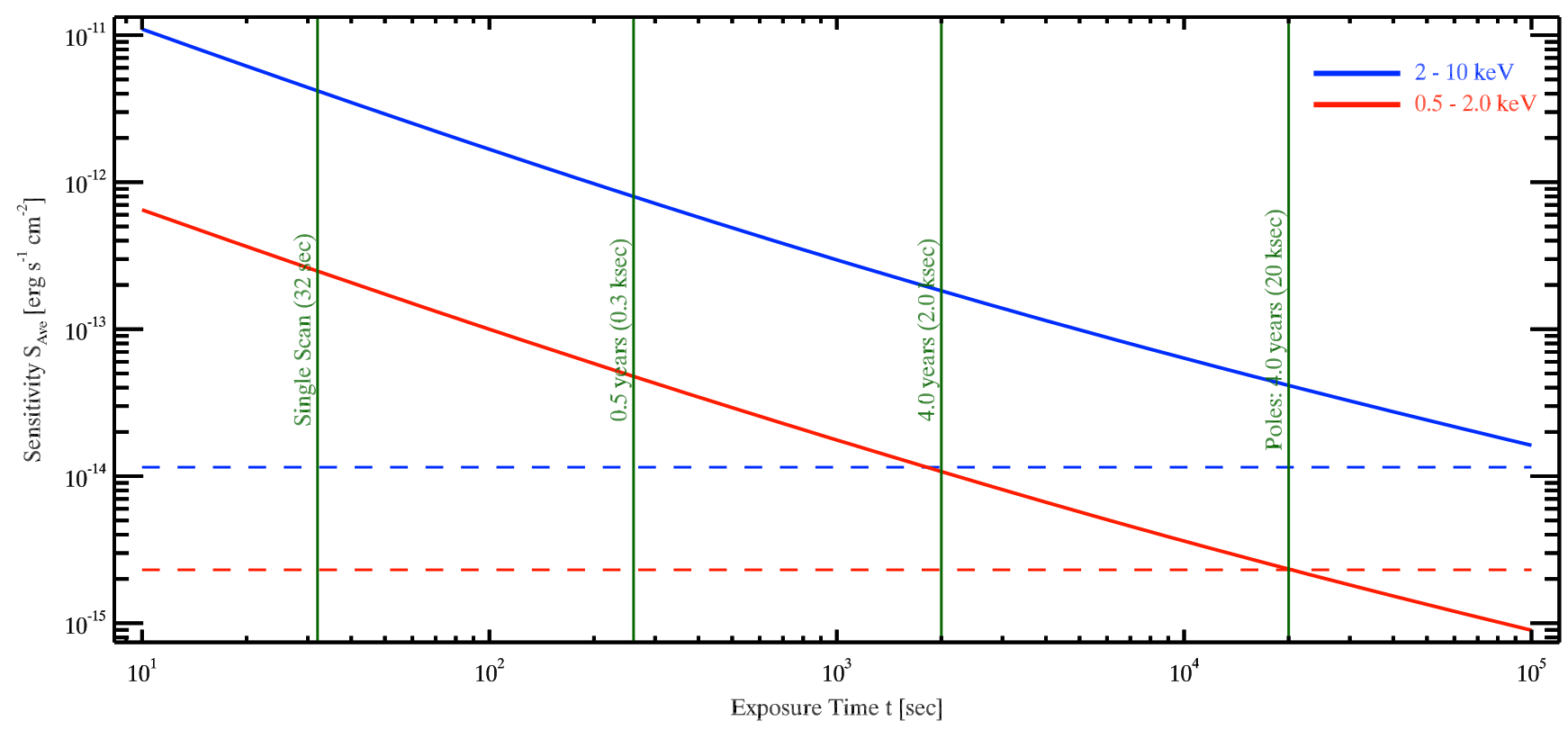

Fig. 1. Average point-source detection sensitivity of the eROSITA telescope as a function of the exposure time in the soft (red) and hard (blue) energy bands for the extragalactic sky. The horizontal dashed lines are the confusion limits for one source per 40 telescope beams (PSF HEW). The vertical line on the left shows the exposure time for a single scan at $100 \%$ observing efficiency, the other vertical lines indicate the average exposure times for different survey durations and at the ecliptic poles (for the soft-band confusion limit) at $80 \%$ observing efficiency.

Table 1. Predicted background count rates.

\begin{tabular}{lcc}
\hline \hline Energy band $[\mathrm{keV}]$ & $0.5-2.0$ & $2-10$ \\
\hline Particle & 0.3 & 2.6 \\
Galactic & 1.8 & 0.0 \\
Extragalactic & 1.9 & 0.5 \\
\hline Total & 4.0 & 3.1 \\
\hline
\end{tabular}

Notes. The count rates are given in units of $10^{-4} \mathrm{cts} \mathrm{s}^{-1}$ per PSF HEW. The extragalactic component accounts for unresolved sources only and at the average four-year survey sensitivity (Table 2).

$\approx 2.17$ PSPC cts s$^{-1} \mathrm{deg}^{-2}$. Variations of the background count rate in this range amount to $\sim 30 \%$ variations in the sensitivity.

The contributions of different background components are summarized in Table 1 . They are consistent within $\approx 10 \%$ with the numbers in the SB. The difference in the soft band comes from the slight difference in the normalization of the extragalactic component. The change in the hard band appears because we used the results of the updated particle background calculations of Perinati et al. (2012) instead of those of Tenzer et al. (2010) which were used in the SB.

\subsection{Average exposure and sensitivity}

With the average background count rates we computed the pointsource detection sensitivity of eROSITA as a function of the exposure time, which is shown in Fig. 1. In this computation we assumed a Poissonian distribution of counts and demanded no more than 200 false point-source detections for the entire sky. This corresponds to one false detection in $\approx 250$ fields of view $\left(\approx 210 \mathrm{deg}^{2}\right)$. For a Gaussian distribution, this falsedetection rate is equivalent to an $\approx 5.0 \sigma$ confidence level in one trial. In converting the count rates into flux we assumed an absorbed power-law spectrum with a photon index $\Gamma=1.9$ and a sky-averaged Galactic absorption of $N_{\mathrm{H}}=6 \times 10^{20} \mathrm{~cm}^{-2}$. We also took into account that only half of the source counts are contained within the PSF HEW.

The sky-averaged exposure time of the survey is

$t_{\text {exp }} \approx 2.0\left(\frac{t_{\text {survey }}}{4 \text { years }}\right)\left(\frac{f_{\text {eff }}}{80 \%}\right)\left(\frac{\text { FOV }}{0.833 \mathrm{deg}^{2}}\right) \mathrm{ks}$,

where $t_{\text {survey }}$ is the survey duration and $f_{\text {eff }}$ is the observing efficiency, whose expected value is $f_{\text {eff }} \approx 80 \%$, and the eROSITA field-of-view (FOV) is $1.03^{\circ}$ in diameter (see the SB). The average numbers of background counts to be accumulated within the PSF HEW in the course of the four-years survey (average exposure time of $\approx 2.0 \mathrm{ks}$ ) are $\approx 0.8$ and $\approx 0.6$ in the soft and hard band. For these numbers and for the chosen confidence level, the source detection thresholds are $\approx 8$ and $\approx 7$ source counts within the PSF HEW. The corresponding point-source detection sensitivities are $\left\langle S_{\text {lim }}\right\rangle \approx 1.1 \times 10^{-14}$ and $\approx 1.8 \times 10^{-13} \mathrm{erg} \mathrm{s}^{-1} \mathrm{~cm}^{-2}$ in the soft and hard band.

After the first half year of the survey, eROSITA will have scanned the whole sky once. At the averaged exposure time of $\approx 260 \mathrm{~s}$, there will be $\approx 0.1$ background counts per PSF HEW in each energy band and the point-source detection threshold will be $\approx 4$ counts. The point-source detection limits for the half-year survey are $\approx 4.8 \times 10^{-14}$ and $\approx 8.0 \times 10^{-13} \mathrm{erg} \mathrm{s}^{-1} \mathrm{~cm}^{-2}$.

The main characteristics of the full survey and its first half year are summarized in Table 2 . The numbers are generally consistent with the SB. The small differences are related to the differences in the background estimates and the larger PSF size used here for the hard band. We also iteratively calculated the resolved fraction of the extragalactic CXB for sensitivities.

Thus, the eRASS will have on average an 30 times better sensitivity in the soft band than the previous all-sky survey in this band conducted by ROSAT (Voges et al. 1999). On the other hand, its sensitivity is between one to four orders of magnitude lower than that of the deep but much more narrow (some of them pencil-beam) extragalactic X-ray surveys conducted by Chandra 
Table 2. Characteristic average parameters of the eROSITA all-sky survey.

\begin{tabular}{|c|c|c|c|c|c|c|}
\hline \multirow{5}{*}{$\begin{array}{l}\text { Survey duration } \\
\text { Sky region } \\
\text { Solid angle }\left[\mathrm{deg}^{2}\right] \\
\text { Exposure time }[\mathrm{s}] \\
\text { Energy band }[\mathrm{keV}]\end{array}$} & \multicolumn{4}{|c|}{4.0 years } & \multirow{4}{*}{\multicolumn{2}{|c|}{$\begin{array}{c}0.5 \text { years } \\
\text { Extragalactic sky } \\
34100\left(|b|>10^{\circ}\right) \\
260\end{array}$}} \\
\hline & \multirow{3}{*}{\multicolumn{2}{|c|}{$\begin{array}{c}\text { Extragalactic sky } \\
34100\left(|b|>10^{\circ}\right) \\
2000\end{array}$}} & \multirow{3}{*}{\multicolumn{2}{|c|}{$\begin{array}{c}\text { Ecliptic poles } \\
90 \\
20000\end{array}$}} & & \\
\hline & & & & & & \\
\hline & & & & & & \\
\hline & $0.5-2.0$ & $2-10$ & $0.5-2.0$ & $2-10$ & $0.5-2.0$ & $2-10$ \\
\hline Resolved extrag. CXB [\%] & 31 & 6 & 53 & 17 & 12 & $\lesssim 1$ \\
\hline Background counts [cts/PSF] & 0.8 & 0.6 & 6.7 & 6.1 & 0.1 & 0.1 \\
\hline Source counts $[\mathrm{cts} / \mathrm{PSF}]$ & 7.6 & 6.8 & 16.5 & 15.6 & 4.4 & 3.9 \\
\hline Sensitivity $\left\langle S_{\lim }\right\rangle\left[10^{-14} \mathrm{erg} \mathrm{s}^{-1} \mathrm{~cm}^{-2}\right]$ & 1.1 & 18 & 0.23 & 4.2 & 4.8 & 80 \\
\hline Source density $\left[\mathrm{deg}^{-2}\right]$ & 84 & 3.7 & 450 & 37 & 10.0 & 0.4 \\
\hline Number of sources $\left[\times 10^{3}\right]$ & 2900 & 130 & 41 & 3.4 & 340 & 13 \\
\hline
\end{tabular}

and XMM-Newton, such as CDFs, COSMOS, Bootes, Lockman Hole, and ChaMP (see Brandt \& Hasinger 2005, for a review).

\subsection{Confusion limit}

For the purpose of this study we assumed that the source confusion becomes important at a source density of one source per 40 telescope beams (=PSF HEW), which for the angular resolution of eROSITA corresponds to a source density of $\approx 460$ and $\approx 260$ sources $\mathrm{deg}^{-2}$ in the soft and hard band. With the average of the extragalactic $\log N-\log S$ curves of G08 and Kim et al. (2007, Table 3, ChaMP+CDFs, hereafter K07), the corresponding flux levels are $\approx 2.3 \times 10^{-15}$ and $\approx 1.2 \times 10^{-14} \mathrm{erg} \mathrm{s}^{-1} \mathrm{~cm}^{-2}$. In the soft band the confusion limit is achieved at an exposure time of $\approx 20 \mathrm{ks}$. In the hard band, source confusion, achieved at an exposure time of $\approx 190 \mathrm{ks}$, is not a problem for eRASS.

\subsection{Sensitivity map}

Owing to the properties of the scan pattern, the two ecliptic poles of eROSITA will have a significantly higher exposure time than the sky on average. This leads to a higher sensitivity at the ecliptic poles. The scan strategy of eROSITA is still under discussion and different scenarios are still possible, depending on whether the satellite rotation axis is continuously pointing at the Sun or moves around it with a slight offset. In the latter scenario, the ecliptic pole regions will occupy a larger solid angle and will be less overexposed. We consider here the extreme case of the continuous Sun-pointing of the scan axis.

Using the exposure map of the four-year survey (Robrade, priv. comm.), we defined the two sky regions at the ecliptic poles of eROSITA, where the exposure time (reduced by the observing efficiency) exceeds the confusion limit of $20.0 \mathrm{ks}$. These two pole regions combined cover a solid angle of $\approx 90 \mathrm{deg}^{2}$. The point-source detection sensitivity in the soft band in the pole regions is defined by the confusion limit and is approximately $2.3 \times 10^{-15} \mathrm{erg} \mathrm{s}^{-1} \mathrm{~cm}^{-2}$, taking into account $\approx 50 \%$ resolved $\mathrm{CXB}$ fraction. The survey characteristics for the pole regions are summarized in Table 2. In the hard band, the confusion limit is reached at a much longer exposure time of $\approx 190 \mathrm{ks}$ and is not relevant for the all-sky survey. The actual hard-band sensitivity in the pole regions is determined by the particular scan pattern. For reference, we list in the Table 2 the sensitivity that can be achieved in the hard band assuming the $20.0 \mathrm{ks}$ exposure.

Outside the poles, the exposure time still varies significantly, with lowest value of $\approx 1.6 \mathrm{ks}$ achieved in the equatorial regions.
These variations will lead to variations of the point-source detection sensitivity across the sky. To compute a realistic sensitivity map of the survey, we took into account variations of the Galactic absorption across the sky along with the exposure map. To this end we used the $N_{\mathrm{H}}$-map of Kalberla et al. (2005). We excluded the Galactic plane and only considered the sky at Galactic latitudes $|b|>10^{\circ}$ for the subsequent calculations. In computing the exposure map we assumed an observing efficiency of $f_{\text {eff }}=80 \%$ and set overexposed regions at the Galactic poles to $20.0 \mathrm{ks}$. The solid angle of this extragalactic sky is $\Omega \approx 34100 \mathrm{deg}^{2}$, which corresponds to $\approx 83 \%$ of the total sky. For the extragalactic sky the arithmetic mean of the exposure map is $\approx 2.1 \mathrm{ks}$, which is close to the average exposure time computed from Eq. (1).

For background calculations, we assumed a constant count rate for the particle background. We assumed that the soft Galactic background is produced in the Local Bubble and therefore is not subject to Galactic absorption, whereas the contribution of the extragalactic CXB component was computed with the $N_{\mathrm{H}}$-map taking into account. The resolved extragalactic CXB fraction was fixed at the sky-average value (see Sect. 2.1 and Table 2). We ignored intrinsic variations of the Galactic and extragalactic $\mathrm{CXB}$, which are unrelated to Galactic absorption. The amplitude of their variations across the sky can be inferred from the ROSAT diffuse sky maps, as described in Sect. 2.1. Background count-rate variations of such amplitude will result in sensitivity variations of $\sim 30 \%$. A part of these variations is caused by the variations of the Galactic absorption and is included in our calculations through the $N_{\mathrm{H}}$-map.

With these assumptions we computed sensitivity maps for the two bands; the one for the soft band is shown in Fig. 2. The sensitivity - solid angle dependences for both bands are shown in Fig. 3.

\section{AGN number counts}

To estimate the source densities and the total numbers of sources detected in different bands we used the source counts results of K07 and G08, displayed in Fig. 4. For K07, we used the bestfit parameters for the ChaMP+CDFs data from their Table 3 and converted the break flux $\left(S_{\mathrm{b}}\right)$ into the hard band from $2.0-8.0 \mathrm{keV}$ to $2.0-10.0 \mathrm{keV}$ assuming a power-law spectrum with a photon index of $\Gamma=1.4$, as was used by K07. The best-fit parameters of K07 and G08 agree well (within $2 \sigma$ ). The difference in number counts between the two $\log N-\log S$ curves is mostly below $10 \%$, the strongest deviation is about $20 \%$ in the flux range of interest (defined by the characteristic sensitivities, 
A. Kolodzig et al.: AGN and QSOs in the eROSITA All-Sky Survey. I.

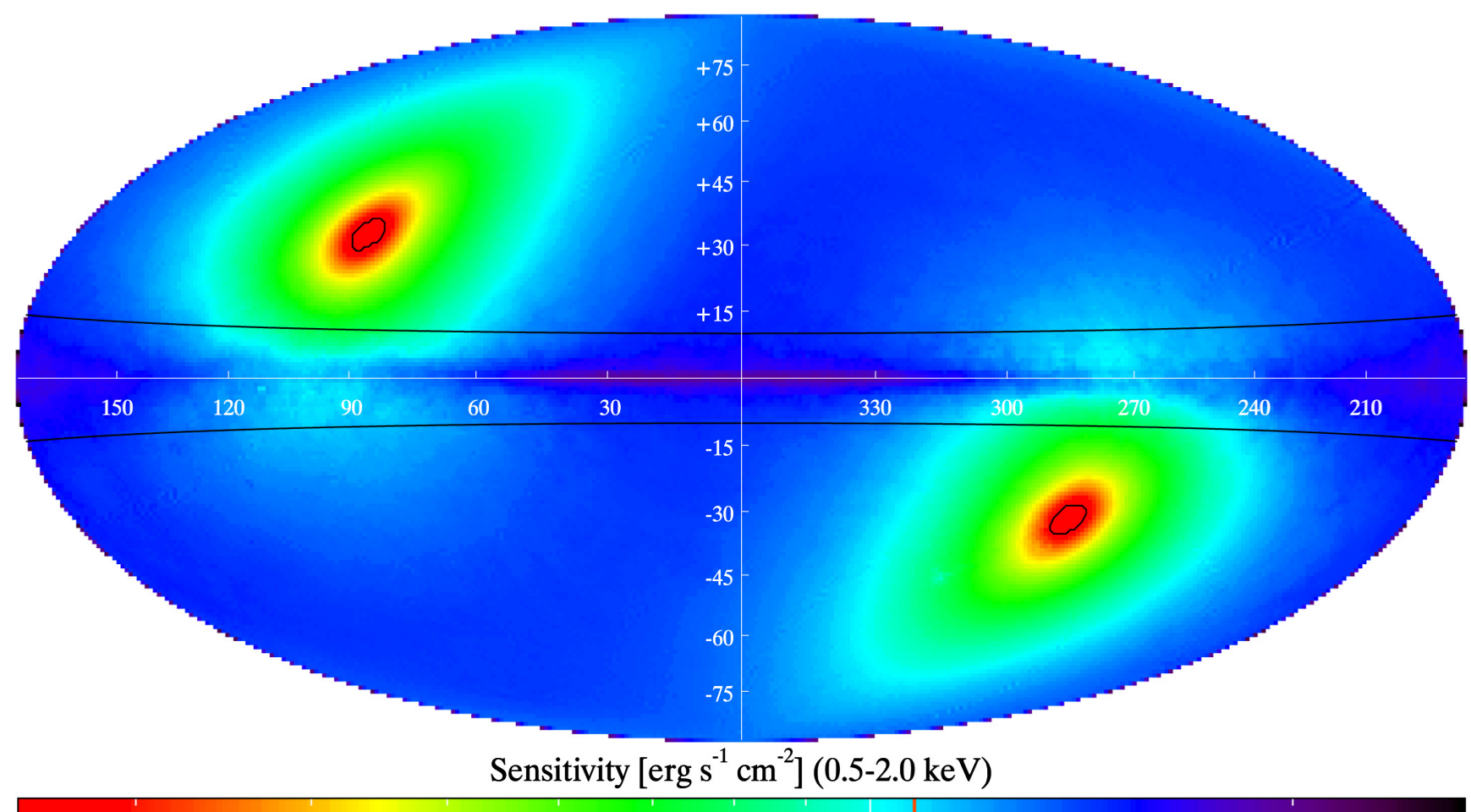

$10^{-14}$

Fig. 2. Four-year soft-band sensitivity map of eRASS in Galactic coordinates $(l, b)$, based on the exposure time map of J. Robrade (priv. comm.) for a continuous Sun-pointing and based on the $N_{\mathrm{H}}$-map of Kalberla et al. (2005). The two black horizontal curves enclose the Galactic plane $\left(|b|<10^{\circ}\right)$, which is excluded from our calculation, and the two regions encircled by black curves are our defined ecliptic poles, where the exposure time was set to $20.0 \mathrm{ks}$. The red vertical line in the horizontal color bar shows the average sensitivity (from Table 2).

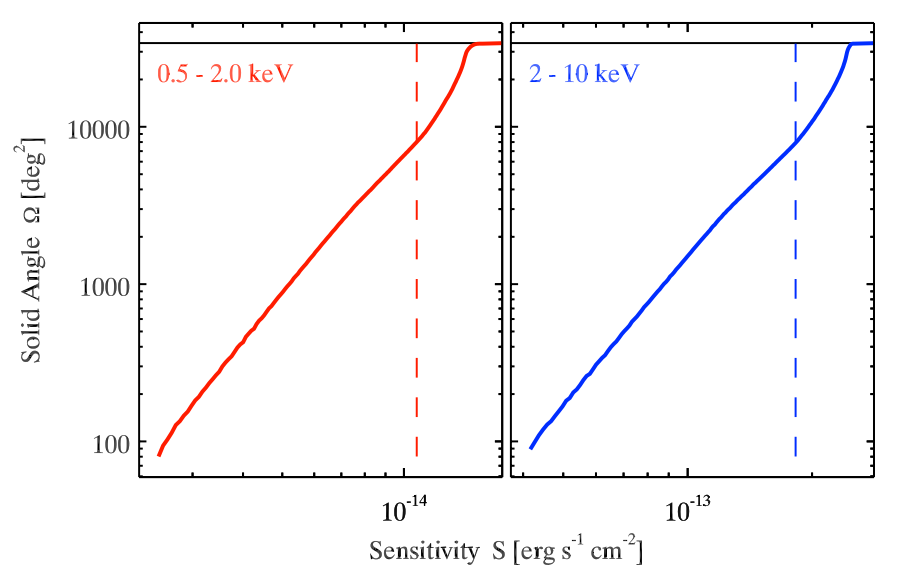

Fig. 3. The sensitivity - solid angle curves for the soft (left panel) and hard (right) bands. The vertical dashed lines show the corresponding average sensitivities from the Table 2 . The horizontal line shows the solid angle of the extragalactic sky.

see Table 2). In the following calculations we used the average of the values given by the two $\log N-\log S$ curves.

With these curves and our sensitivity map in each energy band, we computed the number density map. The arithmetic mean of this map gives us an average number density of $\approx 81 \mathrm{deg}^{-2}$ and $\approx 3.8 \mathrm{deg}^{-2}$ in the soft and hard band. The total numbers of sources detected are $\approx 2.7 \times 10^{6}$ and $\approx 1.3 \times 10^{5}$ for the extragalactic sky. These values differ slightly from those in Table 2 because the latter were computed using the average sensitivities of the survey. They agree reasonably well with those in the SB. About $10 \%$ of these sources in both bands will be detected after the first half year of the survey.

Taking the corresponding sensitivities from Table 2, we computed a number density of $\approx 450 \mathrm{deg}^{-2}$ and $\approx 37 \mathrm{deg}^{-2}$ in the soft and hard band for the ecliptic poles. This translates into $\approx 41000$ and $\approx 3400$ detected extragalactic point-sources after four years.

About $10 \%$ of the brightest AGN in eRASS will be detected with at least $\approx 38$ and $\approx 30$ counts per PSF HEW (corresponding flux limits $\approx 5.4 \times 10^{-14}$ and $\approx 8.0 \times 10^{-13} \mathrm{erg} \mathrm{s}^{-1} \mathrm{~cm}^{-2}$ ) in the soft and hard band. The faintest $10 \%$ will have approximately 8 and 7 counts per PSF HEW $\left(\approx 1.2 \times 10^{-14}\right.$ and $\approx 2.0 \times 10^{-13} \mathrm{erg} \mathrm{s}^{-1} \mathrm{~cm}^{-2}$ ).

We used the sensitivity map to estimate the numbers of AGN expected to be detected in the Galactic plane, $|b|<10^{\circ}$, and obtained $\approx 4.6 \times 10^{5}$ and $\approx 2.2 \times 10^{4}$ sources. This is a somewhat lower number than predicted using the average source density on the extragalactic sky $\left(\approx 6.0 \times 10^{5}\right.$ and $\left.\approx 2.7 \times 10^{4}\right)$ because the Galactic absorption in the Galactic plane is on average an order of magnitude higher than for the extragalactic sky. The higher Galactic X-ray background, not accounted for in these calculations, will additionally reduce the number of AGN at low Galactic latitudes, and high confusion with Galactic sources will make identifying them more difficult.

Finally, we note that AGN will be the most abundant source in eRASS. In addition, eRASS will detect about $\sim 10^{5}$ galaxy clusters (Predehl et al. 2010), $\sim 2 \times 10^{4}$ normal galaxies (Prokopenko \& Gilfanov 2009), and $\sim 4 \times 10^{5}$ stars (Merloni et al. 2012). 

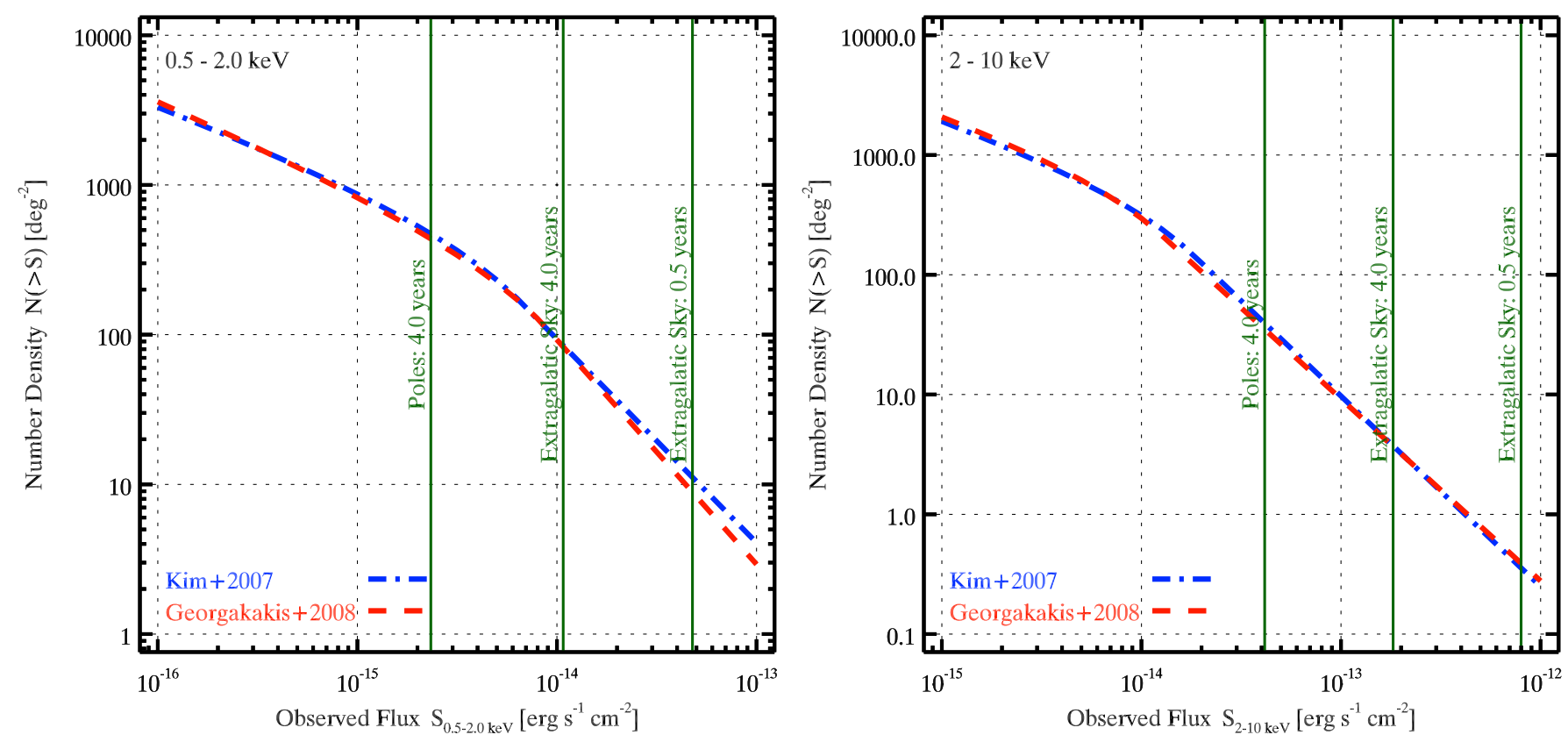

Fig. 4. Cumulative number counts $N(>S)$ for observed X-ray point-sources in the soft $(l e f t)$ and hard band (right). The blue dashed-dotted curve is from Kim et al. (2007, Table 3, ChaMP+CDFs) and the red dashed curve from Georgakakis et al. (2008). The vertical solid lines indicate the point-source detection sensitivity for different survey durations assuming $80 \%$ observing efficiency, and the sensitivity at the 20 ks exposure time, corresponding to the confusion limit in the soft band (left-most line marked "Poles").

\section{X-ray luminosity function of AGN}

With the knowledge of the X-ray luminosity function (XLF) of AGN,

$\phi(L, z)=\frac{\mathrm{d} \Phi(L, z)}{\mathrm{d} \log L}$,

we are able to compute the redshift and luminosity distributions of the AGN expected to be detected in eRASS. The XLF describes the number of AGN $(N)$ per unit co-moving volume $(V)$ and $\operatorname{logarithmic} \mathrm{X}$-ray luminosity $(\log L)$ as a function of $\mathrm{X}$-ray luminosity $L$ and redshift $z$. It is currently believed that the luminosity-dependent density evolution (LDDE) model describes the shape of the observed XLF best (Miyaji et al. 2000; Ueda et al. 2003; Hasinger et al. 2005; Silverman et al. 2008; Ebrero et al. 2009). For completeness, we summarize it below. The LDDE model that parameterizes the AGN XLF has a double power-law

$\phi(L, z)=K_{0}\left[\left(\frac{L}{L_{*}}\right)^{\gamma_{1}}+\left(\frac{L}{L_{*}}\right)^{\gamma_{2}}\right]^{-1} e(L, z)$,

with the density evolution factor given by

$e(L, z)= \begin{cases}(1+z)^{p_{1}} & z \leq z_{\mathrm{c}}(L) \\ \left(1+z_{\mathrm{c}}(L)\right)^{p_{1}}\left(\frac{1+z}{1+z_{\mathrm{c}}(L)}\right)^{p_{2}} & z>z_{\mathrm{c}}(L),\end{cases}$

where the cutoff redshift is given by

$z_{\mathrm{c}}(L)= \begin{cases}z_{\mathrm{c}, 0}\left(\frac{L}{L_{\alpha}}\right)^{\alpha} & L \leq L_{\alpha} \\ z_{\mathrm{c}, 0} & L>L_{\alpha} .\end{cases}$

This LDDE model has nine parameters. Miyaji et al. (2000) defined the density evolution factor (Eqs. (4) and (5)) for the softband XLF in a slightly different way, but the concept remains the same. Hasinger et al. (2005) used the luminosity-dependent density evolution indices $\left(p_{1}\right.$ and $\left.p_{2}\right)$

$$
\begin{aligned}
& p_{1}(L)=p_{1_{44}}+\beta_{1}(\log L-44.0) \\
& p_{2}(L)=p_{2_{44}}+\beta_{2}(\log L-44.0),
\end{aligned}
$$

and therefore the number of parameters increases with the two additional parameters $\left(\beta_{1}\right.$ and $\left.\beta_{2}\right)$ to eleven.

As our default XLF models we used the LDDE model of Hasinger et al. (2005, Table 5, hereafter H05) for the soft band and of Aird et al. (2010, hereafter A10) for the hard band. For the hard-band XLF we used the best-fit model from A10, the "color preselected sample" (their Table 4), which is expected to provide a more accurate description of the XLF at higher redshifts. The parameters of the chosen XLF models are summarized in Table 3.

Based on the XMM-Newton data, Brusa et al. (2009) demonstrated that the soft-band XLF of H05 overpredicts the numbers of high-redshift objects, $z>3$, detected in the COSMOS survey. These authors proposed to introduce an exponential redshift cutoff of the XLF for $z>2.7$,

$\phi=\phi_{\mathrm{H} 05}(z=2.7) \times 10^{0.43(2.7-z)}, \quad z>2.7$,

and showed that with this modification, the observed number counts of high-redshift AGN are reproduced much better. This result was also confirmed by Civano et al. (2011), who used additional Chandra data on the same field and analyzed an $\approx 50 \%$ larger AGN sample than Brusa et al. (2009), and by Hiroi et al. (2012), who analyzed 30 high-redshift $(z>3)$ AGN in the Subaru/XMM-Newton Deep Survey field. Introducing the redshift cutoff results in an insignificant decrease of $\sim 1 \%$ in the total number of AGN above the eRASS sensitivity limit. However, it has a strong effect on the numbers of high-redshift objects, which we discuss in Sect. 5.4. For our default XLF in the soft band, we included the high-redshift cutoff described by Eq. (8), but additionally show results without cutoff. 
Table 3. Parameters of the LDDE model used to compute the luminosity and redshift distributions of the detected AGN.

\begin{tabular}{lcc}
\hline \hline Energy band $[\mathrm{keV}]$ & $0.5-2.0$ & $2-10$ \\
XLF & H05 (Table 5) & A10 (Table 4) \\
\hline$K_{44} / K_{0}^{a}$ & $2.62 \pm 0.16^{b}$ & $8.32 \pm 1.15$ \\
$\log L_{*}{ }^{c}$ & $43.94 \pm 0.11$ & $44.42 \pm 0.04$ \\
$\gamma_{1}$ & $0.87 \pm 0.10$ & $0.77 \pm 0.01$ \\
$\gamma_{2}$ & $2.57 \pm 0.16$ & $2.80 \pm 0.12$ \\
$p_{1_{44}} / p_{1}$ & $4.7 \pm 0.3^{d}$ & $4.64 \pm 0.24$ \\
$p_{2_{44}} / p_{2}$ & $-1.5 \pm 0.7^{d}$ & $-1.69 \pm 0.12$ \\
$z_{\mathrm{c}, 44} / z_{\mathrm{c}, 0}$ & $1.42 \pm 0.11^{e}$ & $1.27 \pm 0.07$ \\
$\log L_{\alpha}{ }_{\alpha}$ & $44.67($ fixed) & $44.70 \pm 0.12$ \\
$\beta_{1}$ & $0.21 \pm 0.04$ & $0.11 \pm 0.01$ \\
$\beta_{2}$ & $0.7 \pm 0.3$ & - \\
\hline
\end{tabular}

Notes. ${ }^{(a)}$ In units of $10^{-7} \mathrm{Mpc}^{-3}$. (b) $K_{0}=K_{44}\left[\left(10^{44.0} / L_{*}\right)^{\gamma_{1}}+\right.$ $\left.\left(10^{44.0} / L_{*}\right)^{\gamma_{2}}\right] \approx 6.69 .{ }^{(c)} \mathrm{erg} \mathrm{s}^{-1} \cdot{ }^{(d)} p_{1}$ and $p_{2}$ are computed from Eqs. (6) and (7). ${ }^{(e)} z_{\mathrm{c}, 0}=z_{\mathrm{c}, 44} 10^{\alpha\left(\log L_{\alpha}-44.0\right)} \approx 1.96$.

As a consistency check, we computed the $\log N-\log S$ distributions based on the chosen XLF models and compared them with the results of the source counts by K07 and G08. The $\log N-\log S$ can be computed by integrating the XLF over luminosity $L$ and redshift $z$ :

$N(>S)=\int_{0}^{z_{\max }} \frac{\mathrm{d} V(z)}{\mathrm{d} z} \int_{\log L_{\min }(S, z)}^{\log L_{\max }} \phi(\log L, z) \mathrm{d} \log L \mathrm{~d} z$.

Here, $\frac{\mathrm{d} V(z)}{\mathrm{d} z}\left[\mathrm{Mpc}^{3} \mathrm{sr}^{-1}\right]$ is the co-moving volume element per redshift and solid angle ${ }^{11}$ and $L_{\min }(S, z)=4 \pi S d_{L}^{2}(z)$, where $d_{L}(z)$ is the luminosity distance (e.g. Hogg 1999). $K$-correction was applied, assuming a power-law spectra with the photon in$\operatorname{dex} \Gamma=1.9$ and no absorption. The same photon index was used to convert the XLFs to the energy bands used in this paper, if the former was determined for a different energy band. It is worth to mention that deep X-ray surveys do not show any evidence of a redshift-dependent photon index (Brandt \& Hasinger 2005).

In Eq. (9) as well as in the calculations described in the next sections, we integrated the XLF model in the luminosity range of $40 \leq \log L\left[\mathrm{erg} \mathrm{s}^{-1}\right] \leq 48$ and in the redshift range of $0 \leq z \leq 7$. Decrease of the $L_{\min }$ in the luminosity integration or increase of the upper limit for the redshift integration, has no significant effect on the number counts $N(>S)$ in our flux range of interest. We note that all experimental XLF determinations are based on AGN samples, that cover a smaller luminosity range, typically $L \geq 10^{42} \mathrm{erg} \mathrm{s}^{-1}$, and a smaller redshift range $\left(z_{\max } \approx 3-5\right)$. Hence, our calculations involve some extrapolation of the measured XLFs to lower luminosities and higher redshift. The uncertainties introduced by this extrapolation are generally small, with a few exceptions that are discussed below.

Using the XLF of H05, we predict a somewhat smaller number of AGN for the soft-band counts than that observed by $\mathrm{K} 07$ and G08, with a strongest deviation of about $30-50 \%$ for the $\log N-\log S$ curve in our flux range of interest. Part of this discrepancy arises because $\mathrm{H} 05$ selected only type $1 \mathrm{AGN}$, and part may be caused by cosmic variance. It is beyond the scope of this work to investigate the origin of this difference in detail, therefore we renormalized the soft-band XLF of H05 upward with a

\footnotetext{
11 The solid angle is converted from steradian to square degrees $\left(\pi^{2} \mathrm{sr}=180^{2} \mathrm{deg}^{2}\right)$.
}

factor of $\approx 1.35$ to match the source counts of K07 and G08 in the flux range of interest. The hard-band $\log N-\log S$ obtained using the XLF of A10 agrees well with the observed source counts, with a strongest deviation of about $3-11 \%$ in the flux range of interest.

\section{Luminosity and redshift distribution of detected AGN}

With the knowledge of the XLF (Sect. 4) we can compute luminosity and redshift distributions of detected AGN as follows:

$$
\begin{aligned}
& \frac{\mathrm{d} N(L)}{\mathrm{d} \log L}=\int_{0}^{z_{\max }(S, L)} \phi(\log L, z) \frac{\mathrm{d} V(z)}{\mathrm{d} z} \mathrm{~d} z \\
& \frac{\mathrm{d} N(z)}{\mathrm{d} z}=\frac{\mathrm{d} V(z)}{\mathrm{d} z} \int_{\log L_{\min }(S, z)}^{\log L_{\max }} \phi(\log L, z) \mathrm{d} \log L,
\end{aligned}
$$

where $z_{\max }$ is defined by the relation $d_{L}\left(z_{\max }\right)=\sqrt{L /(4 \pi S)}$, where $d_{L}\left(z_{\max }\right)$ is the luminosity distance at the redshift $z_{\max }$. For the other quantities and the $K$-correction see the explanation after Eq. (9). The corresponding cumulative distributions are

$$
\begin{aligned}
& N(>L)=\int_{L}^{L_{\max }} \mathrm{d} N\left(L^{\prime}\right) \\
& N(>z)=\int_{z}^{z_{\max }} \mathrm{d} N\left(z^{\prime}\right) .
\end{aligned}
$$

The number of AGN detected in the eRASS as a function of luminosity and redshift is summarized in Fig. 5 and is discussed in more detail in the next two subsections. In computing these distributions we took into account the sensitivity map (Sect. 2.4) of the eRASS via the sensitivity - solid angle distribution shown in Fig. 3. For the overexposed areas at the ecliptic poles we used the sensitivity quoted in Table 2 . The properties of the brightest and faintest $10 \%$ we computed using the flux limits from Sect. 3.

\subsection{Luminosity distribution}

Luminosity distributions of detected AGN are shown in Fig. 6. In the soft band they peak at $\sim 10^{44} \mathrm{erg} \mathrm{s}^{-1}$, with only a small difference between the extragalactic sky sample $\left(\approx 10^{44.0} \mathrm{erg} \mathrm{s}^{-1}\right)$ and the ecliptic poles $\left(\approx 10^{43.8} \mathrm{erg} \mathrm{s}^{-1}\right)$. The peak in the hard band occurs at $\approx 10^{44.4} \mathrm{erg} \mathrm{s}^{-1}$. The median values differ by less than $1 \%$ from the corresponding peak values. Comparing these with the values of $L_{*}$ from the Table 3, the location of the peak is defined by the $L_{*}$ luminosity and does not strongly depend on the survey sensitivity. A change of the latter by two orders of magnitude changes the position of the peak only by $\sim 0.5$ dex. Hence, our predictions for the luminosity distribution are very robust against moderate changes of the survey sensitivity. From the top panel of Fig. 6 one can see that the luminosity distribution in the soft band changes only marginally at high luminosity $\left(\gtrsim 10^{44} \mathrm{erg} \mathrm{s}^{-1}\right)$ if we exclude the high-redshift cutoff of the XLF.

From the cumulative luminosity distributions of the extragalactic sky (right panels of Fig. 6) about 10\% (vertical dashed lines) of the detected AGN will have luminosities higher than $\sim 10^{45} \mathrm{erg} \mathrm{s}^{-1}$. This large sample of luminous AGN $\left(\sim 3 \times 10^{5}\right.$ in 

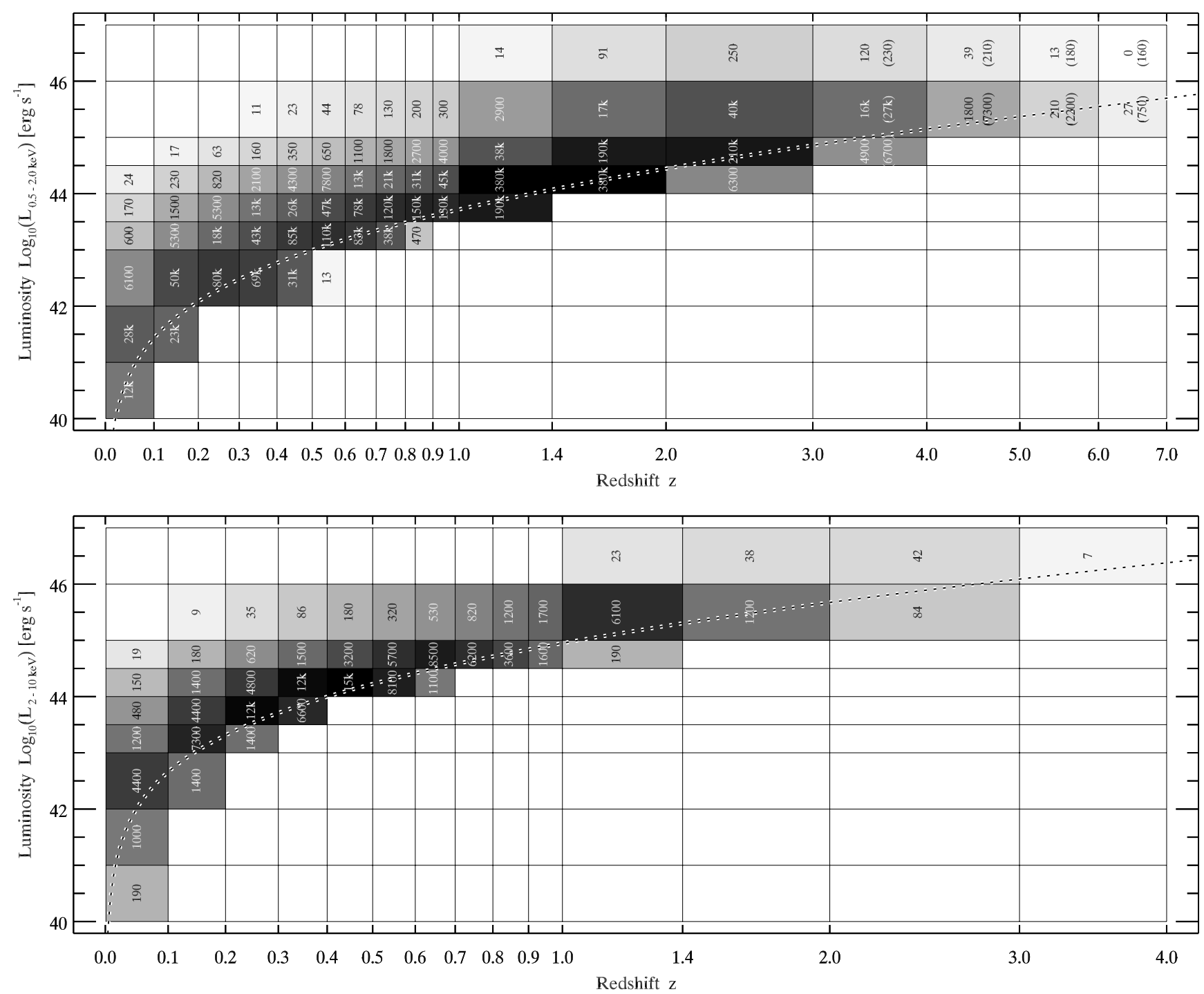

Fig. 5. Number of AGN in different redshift and luminosity bins expected to be detected in the course of the four-year survey in the soft (top) and hard (bottom) bands. White empty bins with no number correspond to zero sources. The dotted line corresponds to the detection limit of eRASS. In the soft-band plot, the numbers in brackets are for the XLF without the exponential redshift cutoff, they are given only if the difference exceeds $10 \%$.

full redshift range) will improve the constraints on the highluminosity end of the XLF. For comparison, the AGN sample of H05 had about 100 AGN with a luminosity higher than $\sim 10^{45} \mathrm{erg} \mathrm{s}^{-1}$.

In the top panel of Fig. 8 the luminosity distribution of the brightest $10 \%$ (those with the highest flux, blue curve) and the faintest $10 \%$ (lowest flux, red) do not differ much from each other and from the distribution of the total sample (black). We note that the luminosity distribution of objects detected in the half-year survey is well represented by that of the brightest $10 \%$ of the sources.

\subsection{Redshift distribution}

Unlike the luminosity distributions, redshift distributions of a flux-limited sample are strongly dependent on the limiting flux (Fig. 7). Correspondingly, the redshift distributions for the extragalactic sky sample and for the poles peak at different redshift, the difference being larger for the hard band. The same is true for the median values, which are listed together with the peak
Table 4. Peak and median values of the redshift distribution of eRASS AGN

\begin{tabular}{lcc|cc}
\hline \hline & \multicolumn{2}{c}{$0.5-2.0 \mathrm{keV}$} & \multicolumn{2}{c}{$2-10 \mathrm{keV}$} \\
& Peak & Median & Peak & Median \\
\hline 4.0 years extragalactic & 0.8 & 1.0 & 0.3 & 0.4 \\
4.0 years ecliptic poles & 1.0 & 1.2 & 0.7 & 0.7 \\
\hline Brightest 10\% & 0.3 & 0.5 & 0.2 & 0.2 \\
Faintest 10\% & 1.1 & 1.2 & 0.5 & 0.5 \\
\hline 0.5 years extragalactic & 0.4 & 0.6 & 0.2 & 0.2 \\
\hline
\end{tabular}

values in Table 4 . The median and peak values in the soft band do not change significantly when we exclude the exponential high-redshift cutoff from our calculations. However, the redshift distribution of AGN at high-redshift does change significantly, which we can see clearly in the top panel of Fig. 7. This is discussed in more detail in Sect. 5.4.

The differential distributions show several breaks that are caused by the derivative discontinuities of the LDDE model. 
A. Kolodzig et al.: AGN and QSOs in the eROSITA All-Sky Survey. I.
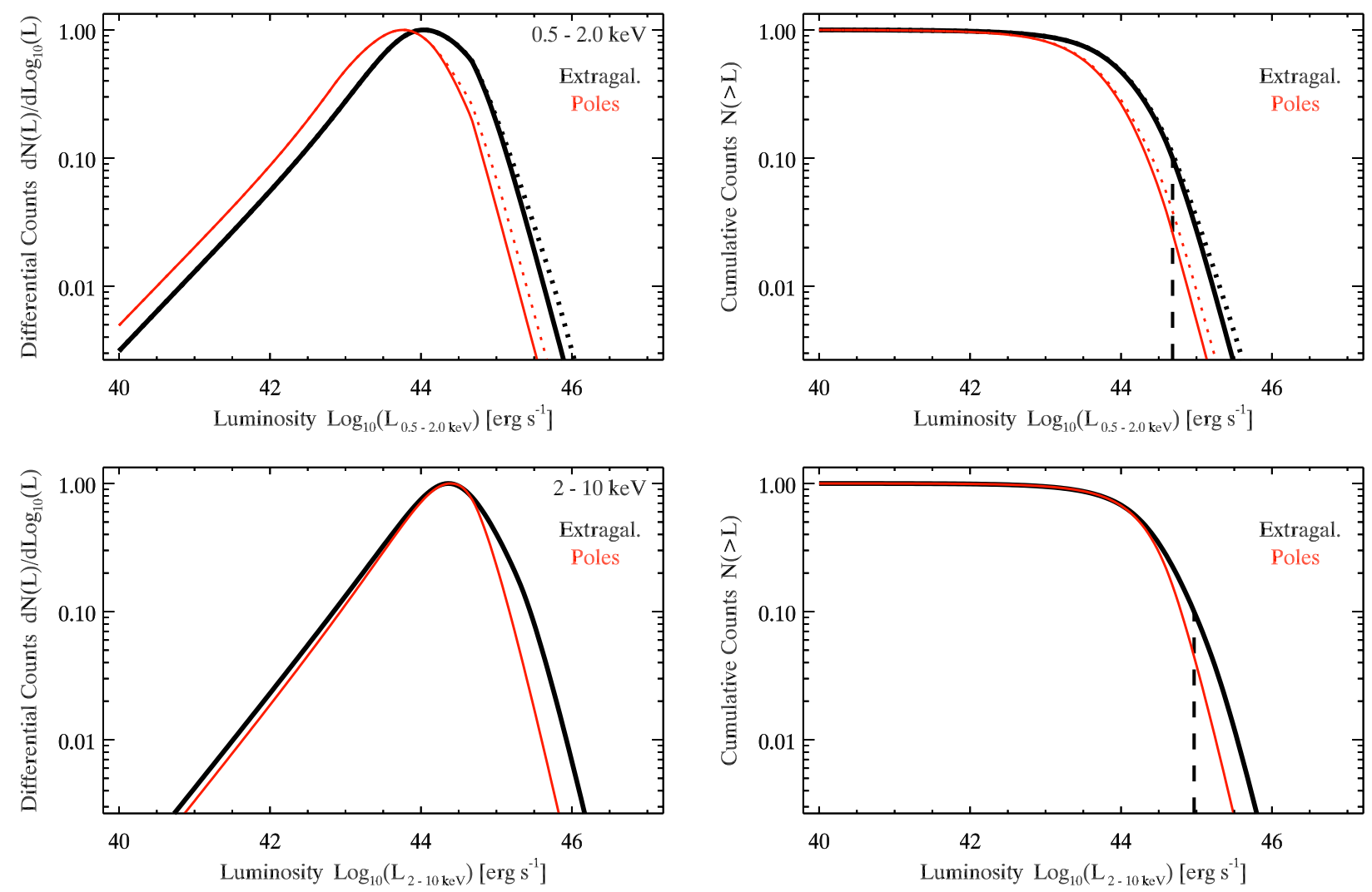

Fig. 6. Differential (left) and cumulative (right) luminosity distribution in the soft (top) and the hard (bottom) band for the four-year eRASS AGN sample in the extragalactic sky (black) and at the ecliptic poles only (red). The distributions are normalized to unity to facilitate comparison of the shapes. The dotted curves in the top panels were computed without the high-redshift cutoff in the soft-band XLF (see Sect. 4). The dashed black vertical lines in the right panels show the luminosity corresponding to the $10 \%$ fraction of sources.
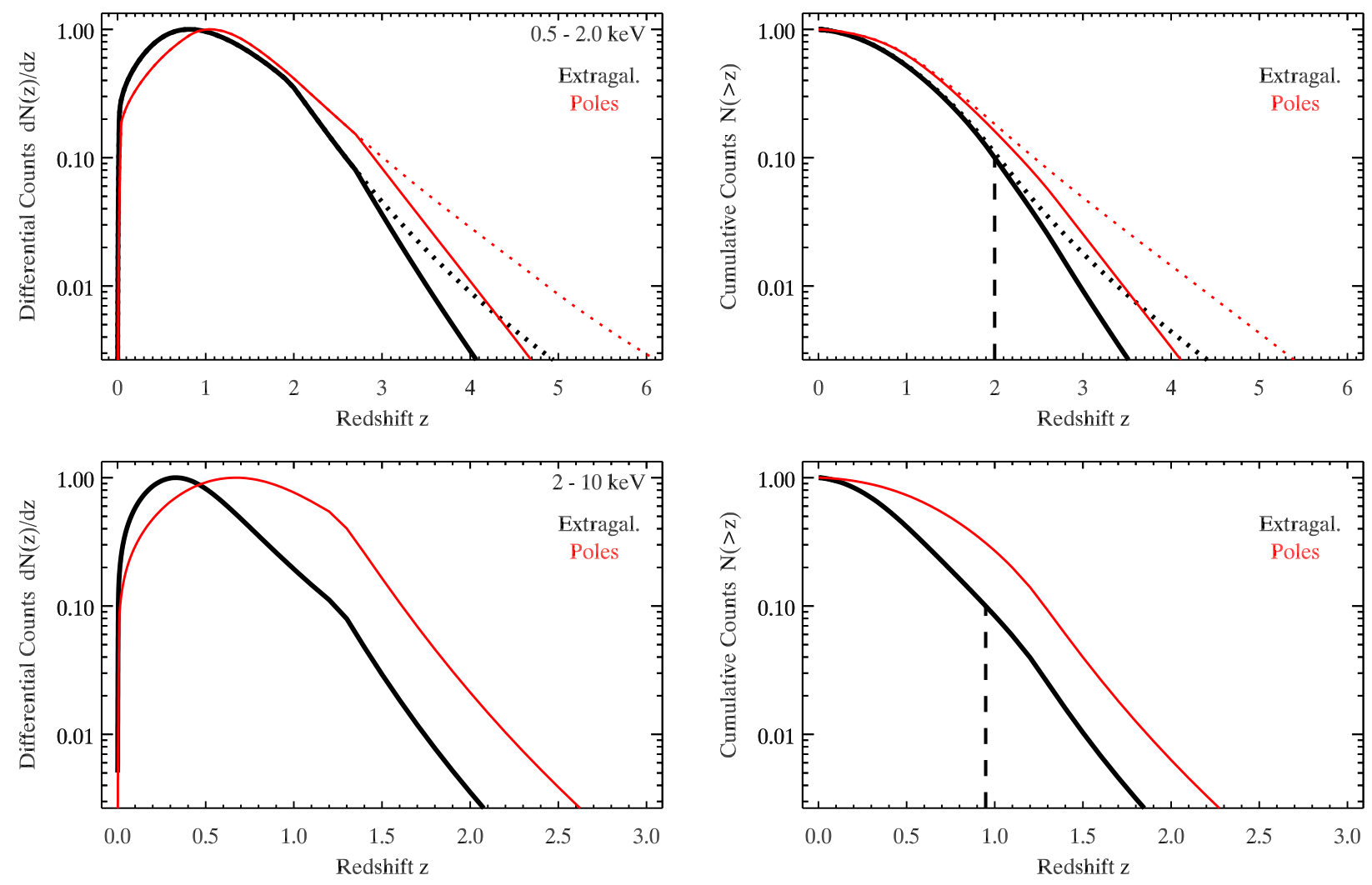

Fig. 7. Same as Fig. 6, but for the redshift distribution. 

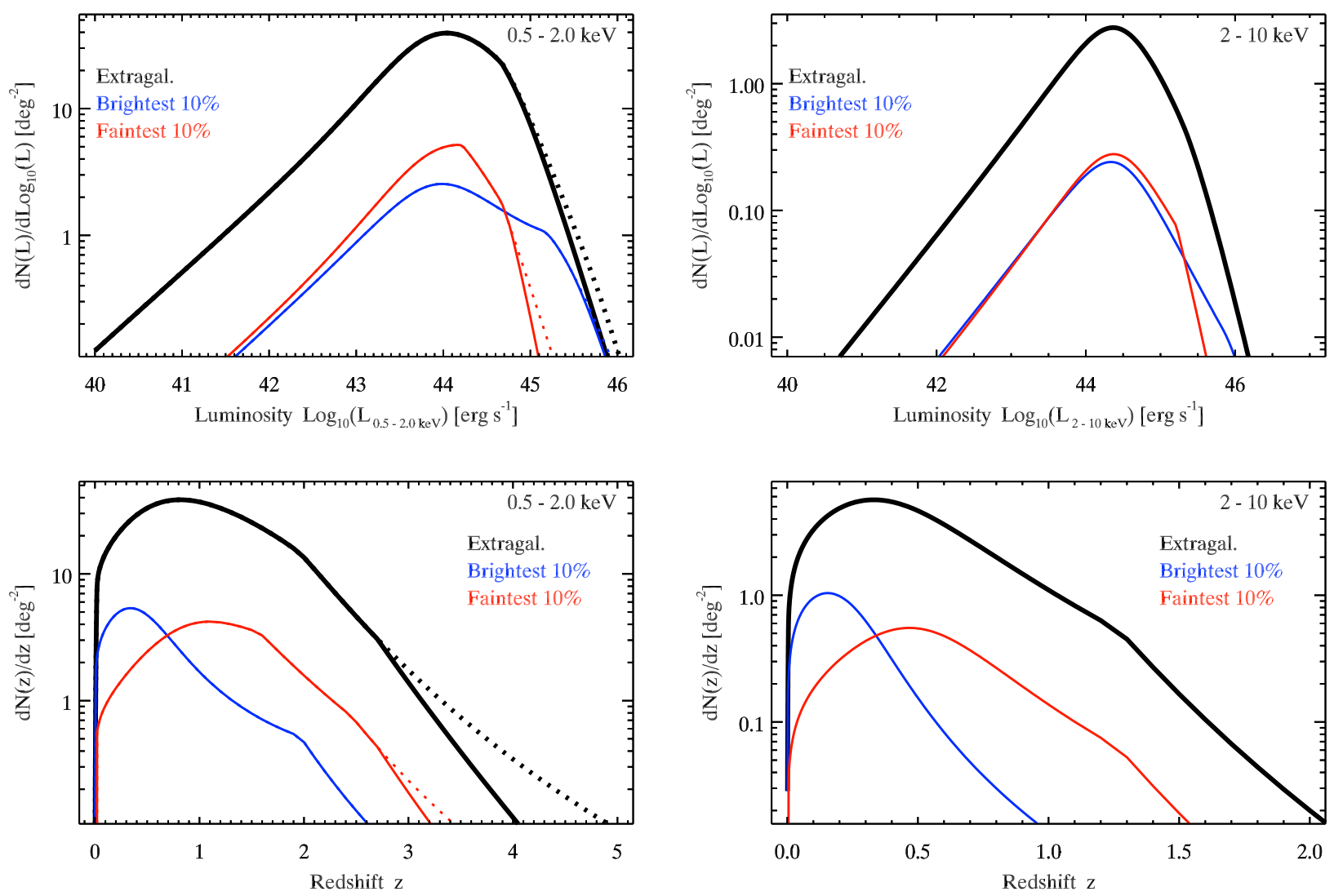

Fig. 8. Differential luminosity (top) and redshift (bottom) distributions in the soft (left) and hard (right) bands for the entire extragalactic AGN sample after fours years (black) and for the brightest 10\% (blue) and faintest 10\% (red). The black curves are same as in Figs. 6 and 7 , but without renormalization to unity.

Another break at the low redshift, $z \sim 0$, appears when the low integration limit in Eq. (11), $\log L_{\min }(S, z)$, becomes equal to the low limit of the interval where the XLF is defined $\left(L=10^{40.0} \mathrm{erg} \mathrm{s}^{-1}\right.$, Sect. 4). These features are not physical and reflect the deficiencies of the functional form used in the LDDE model. However, these deficiencies of the XLF model do not compromise the overall shapes of the redshift (and luminosity) distributions derived in this paper, as long as the overall shape of the AGN X-ray luminosity function is adequately represented by the LDDE model.

In accord with the note made at the beginning of this section, redshift distributions of the brightest and faintest $10 \%$ of the AGN (Fig. 8) peak at significantly different redshifts than the overall distributions (black). Similar to luminosity distributions, the redshift distributions of the objects detected during the half-year survey are similar to the distributions of the brightest $10 \%$. From the cumulative distributions (right panels in Fig. 7.) we conclude that in the soft band, $\approx 50 \%$ of objects in the eRASS sample will be located at $z>1$, whereas $\approx 10 \%$ will be located at $z>2$.

To illustrate the potential of the eRASS AGN sample in the limited sky areas, we show in Fig. 9 the number of objects per redshift bin as a function of redshift for several luminosity groups. For this calculation we chose a sky area of $14000 \mathrm{deg}^{2}$, similar to the area of the Sloan Digital Sky Survey (SDSS), and considered relatively broad redshift bins, consistent with the expected accuracy of photometric redshifts based on the multiband photometry (Salvato et al. 2011). It is obvious from Fig. 9 that even coarse redshift information over relatively limited areas of sky is capable of delivering unprecedented samples of AGN, covering the most luminous AGN ( $>10^{45} \mathrm{erg} \mathrm{s}^{-1}$ ) with an unmatched statistical significance.

\subsection{Uncertainties}

Obviously, the accuracy of our predictions depends on the accuracy of the AGN XLF. This is limited by the moderate numbers of objects used for their construction, typically about 1000 . Although the XLFs obtained by different authors are broadly consistent with each other, there is still a considerable spread between different models. Correspondingly, using XLF models obtained by different authors, we obtained somewhat varying luminosity and redshift distributions of eRASS AGN.

To illustrate the range of uncertainties, we calculated the luminosity and redshift distributions for the soft-band extragalactic sky sample using several different XLF models available in the literature. Along with our default soft-band XLF, we used the XLF of H05 without the exponential redshift cutoff and the XLF models of Miyaji et al. (2000, Table 3) and Ebrero et al. (2009). These XLF models are based on (overlapping) samples, each containing about 1000 objects in total. Because the samples partly overlap, the models are not entirely independent. The resulting luminosity and redshift distributions of the eRASS extragalactic sky sample are shown in Fig. 10. To facilitate the comparison of the shapes, the differential distributions are normalized to unity, whereas the cumulative distributions are shown with their original normalization.

It can be seen from Fig. 10 that different XLFs predict considerably different luminosity and redshift distributions for eRASS AGN. Although the detailed shapes may be not critically 
A. Kolodzig et al.: AGN and QSOs in the eROSITA All-Sky Survey. I.

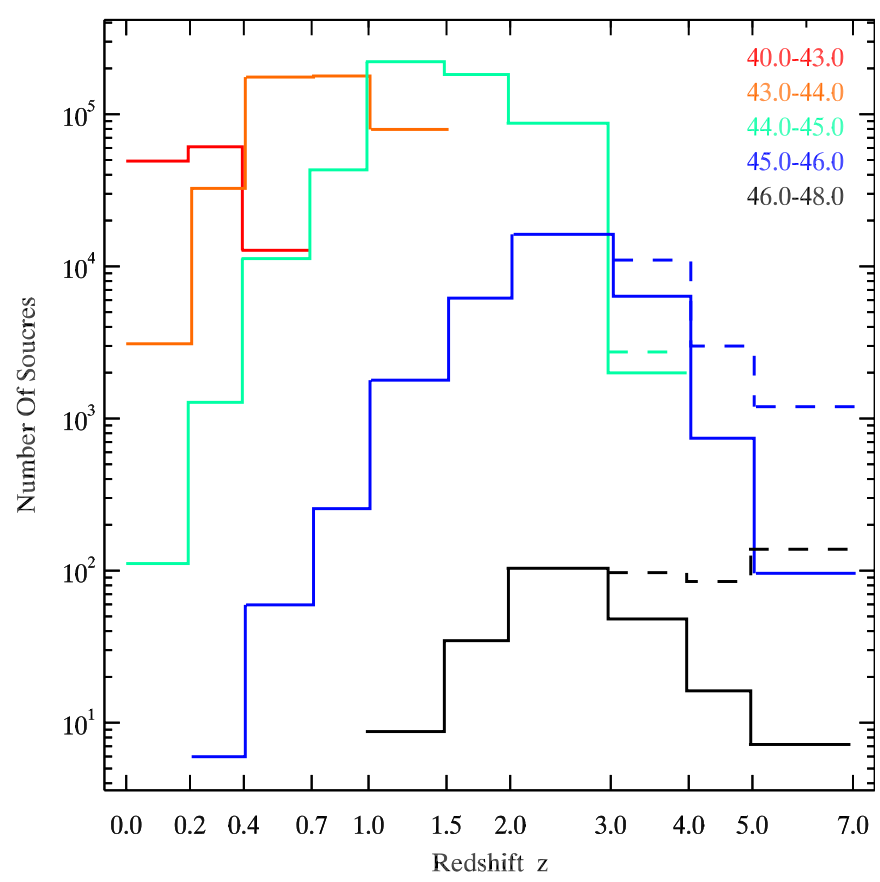

Fig. 9. Number of eRASS AGN in the soft band as a function of the redshift for different luminosity groups in a sky area similar to that covered by SDSS $\left(14000 \mathrm{deg}^{2}\right)$. The solid and dashed histograms show predictions based on our default soft-band XLF with and without exponential high-redshift cutoff (Sect. 4), respectively.

important for the purpose of this paper, median redshifts and luminosities are important characteristics of the eRASS AGN sample. These parameters differ somewhat significantly for the three XLFs, with Miyaji et al. (2000) and Ebrero et al. (2009) presenting two extremes and our default XLF of Hasinger et al. (2005) located in the middle. It is worth mentioning that the primary reason for selecting the XLF of Hasinger et al. (2005) as our default one was the fact that it best reproduced the observed $\log N-\log S$ distributions (Sect. 4). Note also that the total numbers of eRASS AGN are predicted sufficiently accurately from the observed $\log N-\log S$ distributions and therefore are not of significant concern.

In interpreting the plots in Fig. 10 one should keep in mind, that different authors applied different selection criteria in building their samples and used slightly different versions of the LDDE model. In addition, Ebrero et al. (2009) applied a correction for absorption, which the others did not. These differences explain, in particular, the difference in the total AGN surface density predicted by different models. They also explain, at least in part, the considerably large discrepancy in the shapes of the predicted luminosity and redshift distributions. Another part of the difference is probably related to statistical uncertainties in the XLF parameters. Although a detailed comparison of XLFs produced in different studies is beyond the scope of this paper, it would be interesting to see to which extent the discrepancy can be explained by statistical uncertainties. However, the LDDE model is a multiparameter model with a complex correlation pattern between parameters. Therefore a proper error analysis would require knowledge of the covariance matrixes, which are not available anymore (T. Miyaji, priv. comm.). An attempt to treat the XLF parameter errors as independent resulted in unreasonably large uncertainties in the predicted distributions for the eRASS sources. On the other hand, the moderate size of the statistical errors in the observed XLF data in the redshift and luminosity range relevant to the bulk of eRASS AGN ( $L \sim$ $\left.10^{44} \mathrm{erg} \mathrm{s}^{-1}, z \sim 1\right)$ suggests that the behavior of the derived distributions near their peaks is probably not significantly affected by the propagation of statistical errors, therefore the differences seen in Fig. 10 probably reflect genuine differences in XLFs.

Another important factor to be taken into consideration is cosmic variance. Because the AGN XLF determinations rely on the survey that covers only a small fraction of the sky, $\$ 10^{-4}-10^{-3}$ at most, they are subject to cosmic variance. The amplitude of this uncertainty is probably in the $\sim 10 \%$ range (Aird et al. 2010). Obviously, the eRASS sample will provide means for studying this effect in full detail.

We emphasize that we did not consider any separation between different types of AGN. H05 only considered type 1 AGN for their XLF model. If we take into accout the expected small fraction $\left(\sim 10 \%{ }^{12}\right.$, see also Merloni et al. 2012) of type 2 AGN (intrinsic $N_{\mathrm{H}}>10^{21} \mathrm{~cm}^{-2}$ ) and the fairly similar XLF of both types (e.g. Burlon et al. 2011), we expect that the introduced uncertainties will be relatively small.

Finally, we did not take into account the Eddington bias, neither did we consider the details of the source detection and background subtraction algorithms, which will affect to some extent the numbers of detected sources and their $\log N-\log S$ distributions at the faint end. They will also affect the completeness characteristics of the eRASS AGN sample, which will have to be accounted for in constructing XLFs. These are typical properties of flux-limited surveys, especially those conducted in the photon-counting regime, in the limit of small numbers of counts, where the character of the Poissonian distribution of counts manifests itself strongly. The data analysis methods and techniques used to deal with these effects are well known and constitute the standard set of tools in X-ray astronomy. A detailed account of these effects and others (e.g. confusion with extended sources) is beyond the scope of this paper.

\subsection{High-redshift AGN}

The density of AGN at high-redshifts is of particular interest because it can place constraints on the formation scenarios of first supermassive black holes and, hence, on cosmological models (Brandt \& Hasinger 2005). Their numbers in the existing surveys, including those used to produce the XLF models, are very limited. Indeed, the highest redshift bin in the AGN sample of H05 was located at $z=3.2-4.8$ and contained 17 objects. The sample of Miyaji et al. (2000) contained 25 AGN in a somewhat wider redshift interval of 2.3-4.6, and the sample of Ebrero et al. (2009) had no AGN with $z>3$. Moreover, these samples are not entirely independent because they were obtained from overlapping sets of deep surveys. There is only a handful of $z>5$ AGN currently known in X-rays (e.g. Civano et al. 2011). Due to low numbers of distant AGN, the XLF at high redshifts is poorly constrained. As demonstrated below, eRASS will significantly enhance the statistics of high-redshift objects.

Our poor knowledge of the AGN XLF at high-redshifts limits our ability to accurately predict numbers of high-redshift AGN in the eRASS. To estimate the range of uncertainties we calculated their numbers in the soft band using several different XLF models. The resulting cumulative number counts are shown in Fig. 11. For the purpose of this comparison, the curves were rescaled to reproduce the same number density of AGN as the arithmetic mean of our number density map introduced in Sect. 3. The correction factors in the soft band are 1.33 and 1.32

12 http://www.bo.astro.it/ gilli/counts.html 

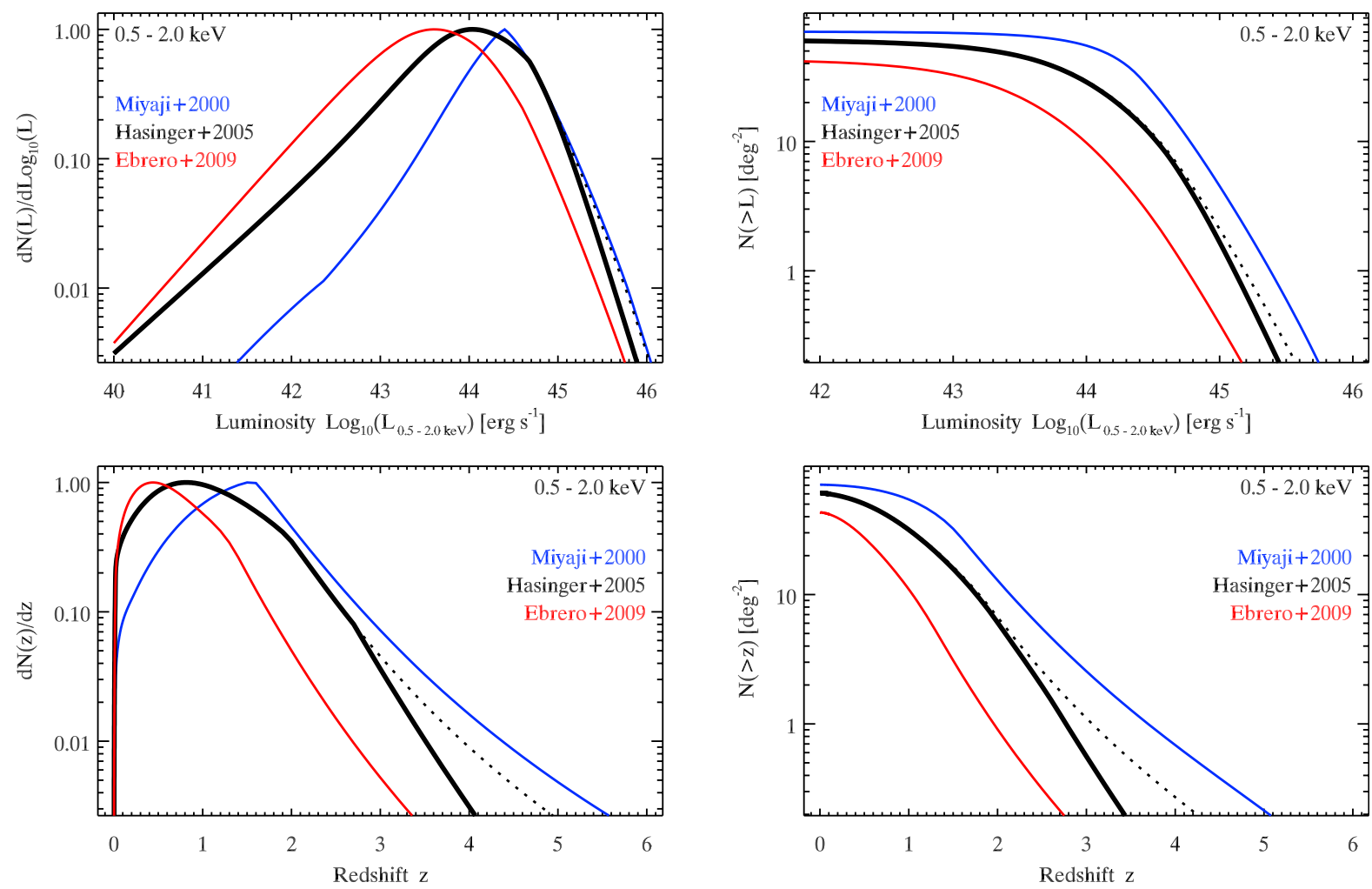

Fig. 10. Differential (left) and cumulative (right) luminosity (top) and redshift (bottom) distributions of the soft-band extragalactic sky sample computed using different XLFs. The thick solid black curves show predictions based on the default XLF model. Also shown are predictions for the XLF model of Miyaji et al. (2000, Table 3), Hasinger et al. (2005, Table 5, without the exponential redshift cutoff, dotted curve), and Ebrero et al. (2009). To facilitate the comparison of shapes, differential distributions in left panels are normalized to unity.

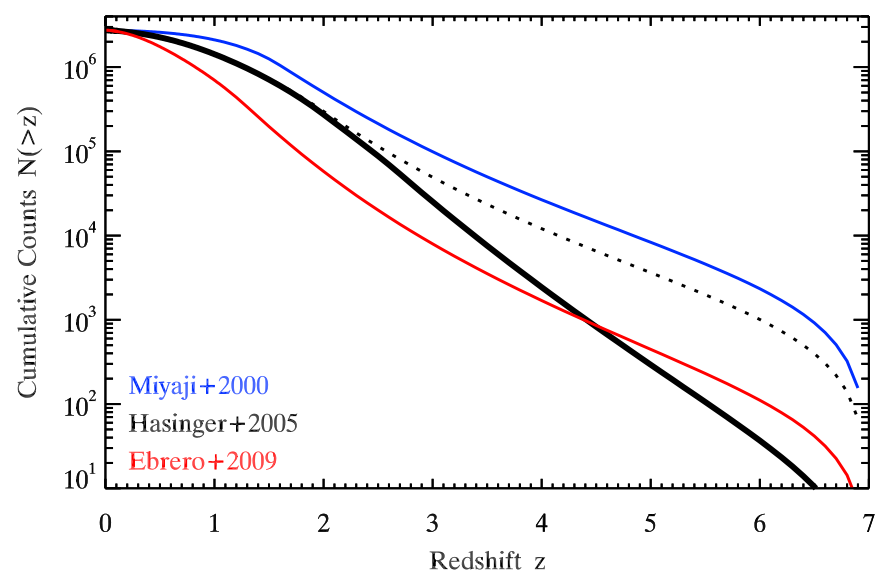

Fig. 11. Numbers of high-redshift AGN, $N(>z)$, expected in the soft band for the extragalactic sky after four years. The thick solid black curve shows the prediction based on the default XLF model. Also shown are predictions for the XLF model of Miyaji et al. (2000, Table 3), Hasinger et al. (2005, Table 5, without the exponential redshift cutoff, dotted curve), and Ebrero et al. (2009). To obtain these curves we integrated the XLFs to the highest redshift of $z=7$. All curves are rescaled to match the average source density computed with our default model (Sect. 3).

for H05 with and without redshift cutoff, respectively, 1.15 for Miyaji et al. (2000, Table 3) and 1.88 for Ebrero et al. (2009).

As one can see from the Fig. 11, the number of high-redshift objects in the extragalactic sky sample is uncertain by more than an order of magnitude, different predictions ranging for $z>3$ from $\sim 10^{4}$ to $\sim 10^{5}$. For $z>4$ and $z>5$ the numbers vary from $\sim 2000$ to $\sim 30000$ and from $\sim 300$ to $\sim 9000$. The exponential redshift cutoff of the H05 XLF (cf. solid and dotted black curves) has a significant effect on the numbers of high-redshift sources bringing it close to the prediction based on the XLF of Ebrero et al. (2009, red curve). On the other hand, the prediction based on the XLF of H05 without a cutoff (dotted black curve) is close to that of Miyaji et al. (2000, blue curve). This large discrepancy of different XLF at high-redshift was previously pointed out by Brusa et al. (2011).

For the number of objects in the $z=3-5$ redshift range our predictions vary from $\sim 8000$ to $\sim 90000$, the default XLF giving $~ 30000$ objects; without the exponential redshift cutoff this number is increased by almost a factor of two.

According to our default hard-band XLF, there will be $\sim 4$ detected AGN in the hard band for the redshift of $z \gtrsim 3.5$. However, the discrepancy between different hard-band XLFs is also large, the predictions ranging from $\sim 2$ (Aird et al. 2010, LADE model of Table 4) to $\sim 200$ (La Franca et al. 2005) AGN for $z \gtrsim 3.5$.

The density of high-redshift objects will be higher in the ecliptic poles (Fig. 7 and Table 2). For the default soft-band XLF, there will be 1 high-redshift $(z \gtrsim 5)$ AGN every $\sim 5 \mathrm{deg}^{2}$, which results in $\sim 17$ objects in total. Without the exponential redshift cutoff this number is an order of magnitude larger. For the other rescaled XLFs from Fig. 11 the number of objects varies between $\sim 20$ and $\sim 600$. Obviously, the higher source density and smaller area will facilitate the search for high-redshift objects in the pole regions.

\section{Redshift determination with the iron $\mathrm{K} \alpha$ line}

The strong $\mathrm{K} \alpha$ line of iron at $\approx 6.4 \mathrm{keV}$ in the spectra of AGN in principle opens the possibility of determining redshifts by means 
of X-ray spectroscopy. Below, we investigate this possibility for the parameters of eRASS characteristics of the eROSITA telescope.

We assumed that the continuum spectrum is described by an absorbed power-law with a photon index of $\Gamma=1.9$ and $N_{\mathrm{H}}=6 \times 10^{20} \mathrm{~cm}^{-2}$. The shape of the iron $\mathrm{K} \alpha$ line may be fairly complex and typically includes narrow and broad components, with centroids depending on the ionization state (e.g. $6.4 \mathrm{keV}$ for the neutral component), and may be complicated by other features (e.g. the $7.11 \mathrm{keV}$ absorption edge; e.g. Gilli et al. 1999; Nandra et al. 2007; Corral et al. 2008; Shu et al. 2010; Krumpe et al. 2010; Chaudhary et al. 2012). However, as the final result of this calculation turn out to be somewhat negative, we ignored this complexity and used a simple model ${ }^{13}$, consisting of a single Gaussian line at $6.4 \mathrm{keV}$ with an intrinsic width of $\sigma_{\mathrm{Fe}}=200 \mathrm{eV}$ and an equivalent width of $150 \mathrm{eV}$ (rest-frame values).

To investigate the detectability of the iron $\mathrm{K} \alpha$ line in the spectra of eRASS AGN, we performed the following simulations: we chose a number of flux values in the $10^{-11}-10^{-10} \mathrm{erg} \mathrm{s}^{-1} \mathrm{~cm}^{-2}$ flux range. We fixed the redshift and for each flux value simulated 1000 spectra using the phabs (zpowerlw + zgauss) model in XSPEC (version 12.7.0, Arnaud 1996). Each spectrum was fit with the same model. In the fit, the parameters $\sigma_{\mathrm{Fe}}$ and $N_{\mathrm{H}}$ were fixed, the initial values of other parameters were set at their simulated values. After 1000 spectra were simulated, the distribution of the best values of the redshift was analyzed. It was fit with a Gaussian distribution, then the points outside the $\pm 3 \sigma$ range were marked as catastrophic failures and clipped out, and the distribution was fit by a Gaussian again. The newly obtained width of the Gaussian determines the accuracy of the redshift determination $\sigma_{z}$. The catastrophic error fraction was then recomputed as a fraction of objects outside $\pm 3 \sigma_{z}$.

Our results for the redshift $z=0$ are shown in Fig. 12. As one can see from the plot, even at considerably large number of counts, $\sim 1500$ in the hard band, corresponding to a flux of $\sim 2 \times 10^{-11} \mathrm{erg} \mathrm{s}^{-1} \mathrm{~cm}^{-2}$, the fraction of catastrophic errors is still large, $\sim 40 \%$. This is caused by the steep decrease of the eROSITA efficiency curve with energy, by more than an order of magnitude between 2 and $6 \mathrm{keV}$. As a result, even at large total number of counts, the number of counts at $\sim 6 \mathrm{keV}$, is too small for a reliable line detection in the flux range of interest.

From the middle panel of the Fig. 12, the accuracy of the redshift measurements for the remaining $\sim 60 \%$ of objects may seem to be reasonably good, $\delta z \lesssim 0.05$. Obviously, this is a result of its definition, which relies on excluding catastrophic failures. This definition works well when the there are few catastrophic failures. However, the effect of small $\delta z$ is nullified when the fraction of catastrophic failures is large. Furthermore, the numbers of objects in this flux range is of the order of 100 on the entire extragalactic sky, which is too small to be useful. The majority, if not all of these bright objects, will be previously known AGN with known redshifts.

The increase of the effective area toward low energies could improve the situation at higher redshifts. For a $z \approx 2$ object, for example, the observed energy of the iron $\mathrm{K} \alpha$ line would fall near the peak of the eROSITA sensitivity and would lower the lowest flux required for a reliable redshift determination using the iron $\mathrm{K} \alpha$ line to $\sim 10^{-12} \mathrm{erg} \mathrm{s}^{-1} \mathrm{~cm}^{-2}$. Unfortunately, the relatively small number of $z \approx 2$ objects (a few hundreds) and their

\footnotetext{
13 In the flux range of interest, more complex models generally lead to larger fraction of catastrophic failures.
}

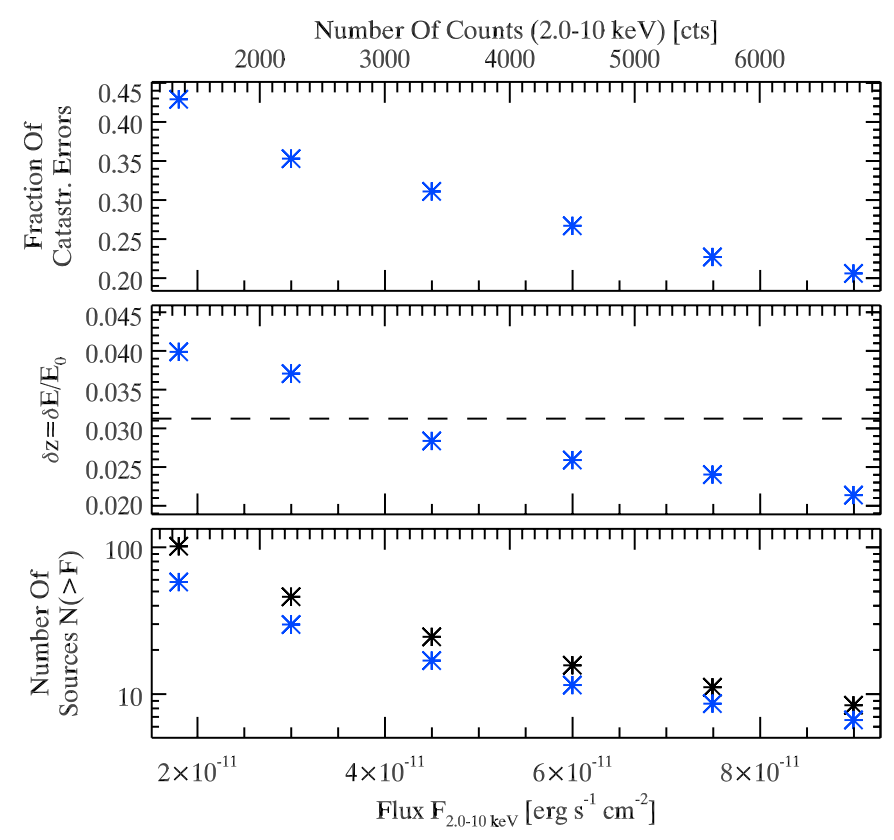

Fig. 12. Feasibility of using the iron $\mathrm{K} \alpha$ line for the redshift determination at $z=0$. See Sect. 6 for details. Top: fraction of catastrophic failures. Middle: accuracy of the redshift determination. The black dashed line corresponds to the energy resolution of eROSITA. Bottom: number of sources for which the redshift can be determined with the accuracy shown in the middle panel, the catastrophic failures excluded (black points show only the $\log N-\log S$ ).

low fluxes negate the advantage given by the larger effective area at low energies. However, we note that a spectral analysis would still be possible for the sources of known redshift, and that one can still use spectral stacking analysis (e.g. Chaudhary et al. 2010) to study the average properties of the iron $\mathrm{K} \alpha$ line of AGN.

\section{Transient events and flux variability}

In the four-year survey, eROSITA will scan the whole sky eight times (one full-sky survey per half year). The telescope rotates around an axis pointing either directly towards the Sun or with some offset (see Sect. 2) and will complete one full circle on the sky every four hours (Predehl et al. 2010). The plane of the scan rotates with an average rate of one degree per day. With this scan geometry, a point on the equator will be scanned $\approx 6$ times every half year, separated by $\approx 4 \mathrm{~h}$. The number of consecutive scans per one survey increases with latitude as $\propto \cos ^{-1}(\delta)$, the poles being scanned continuously every four hours during the entire duration of the survey. This scan pattern defines two different sampling rates: $\Delta t_{1}=0.5$ years and $\Delta t_{2}=4 \mathrm{~h}$, corresponding to frequencies of $\approx 6 \times 10^{-8}$ and $\approx 7 \times 10^{-5} \mathrm{~Hz}$.

For one full-sky survey, the average exposure time is $\approx 320 \mathrm{~s}^{14}$. At this exposure time, 5 counts correspond to the flux of $\approx 2 \times 10^{-14}$ and $\approx 4 \times 10^{-13} \mathrm{erg} \mathrm{s}^{-1} \mathrm{~cm}^{-2}$ in the soft and hard band. These numbers define the sensitivity of eRASS to the events (e.g. flares) occurring on the half-year timescale. With eight measurements it is also possible to estimate the rms variability on the corresponding timescale. To estimate the sensitivity, we took into account that the sample variance is distributed as $\sigma^{2} \chi_{\mathcal{N}-1}^{2} /(\mathcal{N}-1)$, where $\sigma$ is the sample rms, $\mathcal{N}$ is the number

${ }^{14}$ For this calculation it is more appropriate to assume an observing efficiency of $100 \%$. 
of points and $\chi_{\mathcal{N}-1}^{2}$ is the $\chi^{2}$-distribution with $\mathcal{N}-1$ degrees of freedom. Therefore the $1 \sigma$ error of the fractional $\mathrm{rms}^{2} \mathrm{de}-$ termination is $\delta\left(\mathrm{rms}^{2}\right) \approx \sqrt{2 /(\mathcal{N}-1)} \times(S / N)^{-2}$, where $S / N$ is the signal-to-noise ratio. As an estimate of the sensitivityto-source flux variability, we took the square root of this expression and obtained $\delta(\mathrm{rms}) \approx 0.73 \times(S / N)^{-1}$. Thus, for a $2 \times 10^{-13} \mathrm{erg} \mathrm{s}^{-1} \mathrm{~cm}^{-2}$ soft-band source, a fractional variability of rms $\sim 20 \%$ will be detected with a $2 \sigma$ confidence. On the extragalactic sky, about 35000 sources are above this flux threshold.

At the average exposure time in a single scan $(\approx 32 \mathrm{~s})$, 5 counts correspond to a flux of $\approx 2 \times 10^{-13} \mathrm{erg} \mathrm{s}^{-1} \mathrm{~cm}^{-2}$ in the soft band. There are about 39000 sources on the extragalactic sky above this flux level. This also defines the sensitivity of the eRASS to transient events on an approximate timescale of hours. Except for the sources in the polar regions, aperiodic variability on these timescales can be measured only for a small number of sources.

\section{Optical counterparts}

To fully explore the potential of the eRASS, extensive optical coverage will be critical. The primary, but not sole goal of this coverage will be to provide identifications and redshift information. A detailed discussion of the feasibility and possible strategies of the optical support of the eRASS and its synergies with various ongoing and future optical surveys is beyond the scope of this paper and is currently extensively discussied in the eROSITA collaboration. In this section we investigate the expected optical magnitude distribution of the eRASS AGN. To this end, we used results of the XMMCOSMOS (Hasinger et al. 2007) and XBootes (Murray et al. 2005) surveys. For the COSMOS field, we used the results of Brusa et al. (2010), who cross-correlated the original XMMCOSMOS catalog of X-ray sources of Cappelluti et al. (2009) with the data of the optical survey of the COSMOS field by Capak et al. (2007). From these data we selected sources with a $0.5-2.0 \mathrm{keV}$ flux, which exceeds the eRASS four-year detection threshold (214 out of 1710 sources fulfill the condition $S_{0.5-2.0 \mathrm{keV}} \geq 10^{-14} \mathrm{erg} \mathrm{s}^{-1} \mathrm{~cm}^{-2}$ ), which had a high identification reliability (204 out of 214), and were not brighter than $I_{\mathrm{AB}}=14.0 \mathrm{mag}$ (197 out of 204). This selection resulted in a sample of 197 sources for which we obtained the $I$-band AB-magnitude distribution.

We similarly analyzed the XBootes field, cross-correlating the X-ray and optical catalogs for this field (Kenter et al. 2005; Brand et al. 2006). We selected point-like sources (class $\geq 0.50$ ) with $S_{0.5-2.0 \mathrm{keV}} \geq 10^{-14} \mathrm{erg} \mathrm{s}^{-1} \mathrm{~cm}^{-2}$ (565 out of 3213), for which an optical counterpart was found $(\mathrm{St}=1)(565$ out of 565 ) with a high probability of true identification (Popt $\geq 0.95$ ) (561 out of 565) and an optical flux of $I_{\mathrm{AB}}>14.0$ mag (536 out of 561). We thus selected $536 \mathrm{X}$-ray sources, for which we computed the cumulative $I$-band magnitude distribution, converting the Vega magnitudes to AB-magnitudes with the conversion factor from Blanton \& Roweis (2007, Table 1): $m_{\mathrm{AB}}=$ $m_{\mathrm{Vega}}+m_{\mathrm{AB}}($ Vega $)$ with $m_{\mathrm{AB}}($ Vega $)=0.45$ for the $I$-band.

These $I$-band magnitude distributions for the COSMOS and Bootes fields are plotted in the Fig.13. They show a good agreement between the results for the two different fields, meaning that we have a very good knowledge of the expected magnitude distribution of sources at bright X-ray fluxes. Comparing this distribution with the limiting magnitude of the Sloan Digital Sky Survey in the $i$-band, $21.3 \mathrm{mag}$ at the $95 \%$ completeness (Abazajian et al. 2009), we conclude that about $\approx 80 \%$

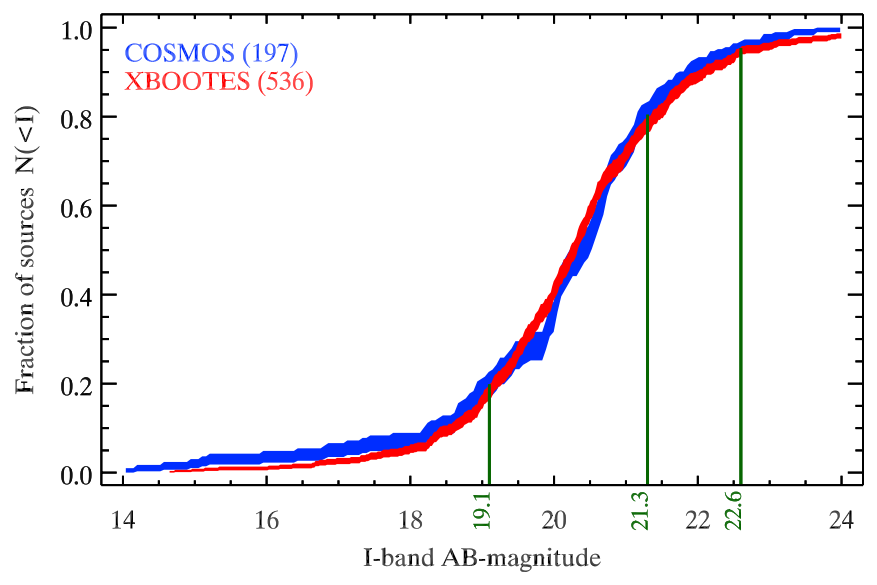

Fig. 13. Cumulative $I$-band AB-magnitude distribution of AGN in the COSMOS and XBOOTES fields with the $0.5-2.0 \mathrm{keV}$ flux exceeding the four-year eRASS detection threshold. The thickness of the curves represents the standard deviation of a binomial distribution. The vertical lines show the photometric sensitivities of the SDSS (21.3 mag) and Pan-STARRS PS1 (22.6 mag) and the magnitude limit for SDSS spectroscopy (19.1 mag).

of the eRASS AGN in the SDSS sky will have optical counterparts. Taking into account the sky area covered by SDSS, $\approx 14500 \mathrm{deg}^{2}$, we estimate that about $\sim 1 / 3$ of eRASS objects will have an optical counterpart in the SDSS photometric cata$\log$. About $\approx 20 \%$ of objects will be brighter than the spectroscopic limit of the SDSS, $i=19.1 \mathrm{mag}$ for quasars at $z<3$ (Richards et al. 2002), that is, some fraction of these objects will have SDSS spectra.

Repeating this analyzsis for the half-year sensitivity of eRASS, we expecte that almost all eRASS AGN in the SDSS sky will have optical counterparts.

One can see from Fig. 13 that approximately $95 \%$ of eRASS AGN will be brighter than $I_{\mathrm{AB}}=22.5 \mathrm{mag}\left(R_{\mathrm{AB}} \approx 23.0 \mathrm{mag}\right)$. The Pan-STARRS PS1 $3 \pi$ survey will exceed this depth with its expected sensitivity of $\approx 22.6$ mag in one visit (Chambers \& the Pan-STARRS team 2006). The three year PS1 sensitivity in the $I$-band will reach $\approx 23.9$ mag and will cover virtually all eRASS objects in the field of the $3 \pi$ survey .

\section{Summary and conclusions}

We computed various statistical characteristics of the expected eRASS AGN sample, including their luminosity- and redshift distributions, and the magnitude distributions of their optical counterparts.

The eROSITA all-sky survey will produce an unprecedented sample of about 3 million X-ray selected AGN. With a median redshift of $z \approx 1$, approximately $40 \%$ of the eRASS objects will be located between redshifts $z=1$ and $z=2$ (Fig. 7). We predict that about $10^{4}-10^{5}$ AGN beyond redshift $z=3$ and about 2000-30 000 AGN beyond redshift $z=4$, the exact numbers depending on the behavior of the AGN XLF in the high-redshift and high-luminosity regimes (Fig. 10).

The eRASS AGN sample will open the possibility of studying the growth of supermassive black holes, their co-evolution with host galaxies and dark matter halos, and their relation to the large-scale structure to unprecedented detail, and potentially, it may also help to constrain cosmological parameters (Kolodzig et al. 2013; Hütsi et al. in prep.). Importantly, it will permit one 
to conduct these studies beyond redshift $z=1$, which is poorly covered by the current optical surveys.

To fully exploit this potential of eRASS, an extensive optical support will be critical. One of the main goals of optical follow-up will be to provide redshifts for eRASS AGN, but its importance will reach far beyond this, including, for example, studies of the co-evolution of supermassive black holes and their host galaxies (see Kolodzig et al. 2013 for a discussion of the different goals of optical follow-ups and their requirements and prospects). With the capabilities of the currently available facilities and their time allocation strategies, measuring of optical spectra for the entire sample of the $\sim 3$ million objects does not appear to be achievable on realistic timescales. However upcoming hardware and survey programs and proposals, for instance, 4MOST (de Jong et al. 2012) and WEAVE (Dalton et al. 2012), can make this task more realistic, especially for some limited areas of sky. Furthermore, introducing multiband photometry and other improvements of the photometric redshift measurement techniques will make determining of photometric redshifts for large samples of eRASS AGN possible (Salvato et al. 2011; Saglia et al. 2012).

Acknowledgements. We thank Jan Robrade for the exposure-time map of eROSITA and additional calculations, Peter Friedrich for the details about the PSF of eROSITA, Thomas Boller for the details about the eROSITA background calculation, Takamitsu Miyaji for discussions of AGN XLF, Andrea Merloni for useful discussions, and Peter Predehl for background information about eROSITA. A. Kolodzig acknowledges support from and participation in the International Max-Planck Research School (IMPRS) on Astrophysics at the Ludwig-Maximilians University of Munich (LMU). M. Brusa acknowledges support from the FP7 Career Integration Grant "eEASy: eROSITA Euclid AGN Synergies" (CIG 321913). The research made use of grant NSh-5603.2012.2 and programs P-19 and OFN-17 of the Russian Academy of Sciences.

\section{References}

Abazajian, K. N., Adelman-McCarthy, J. K., Agüeros, M. A., et al. 2009, ApJS, 182,543

Aird, J., Nandra, K., Laird, E. S., et al. 2010, MNRAS, 401, 2531

Anders, E., \& Grevesse, N. 1989, Geochim. Cosmochim. Acta, 53, 197

Arnaud, K. A. 1996, in Astronomical Data Analysis Software and Systems V, eds. G. H. Jacoby, \& J. Barnes, ASP Conf. Ser., 101, 17

Blanton, M. R., \& Roweis, S. 2007, AJ, 133, 734

Brand, K., Brown, M. J. I., Dey, A., et al. 2006, ApJ, 641, 140

Brandt, W. N., \& Hasinger, G. 2005, ARA\&A, 43, 827

Brusa, M., Comastri, A., Gilli, R., et al. 2009, ApJ, 693, 8

Brusa, M., Civano, F., Comastri, A., et al. 2010, ApJ, 716, 348

Brusa, M., Gilli, R., Civano, F., et al. 2011, Mem. Soc. Astron. Ital. Suppl., 17, 106
Burlon, D., Ajello, M., Greiner, J., et al. 2011, ApJ, 728, 58

Capak, P., Aussel, H., Ajiki, M., et al. 2007, ApJS, 172, 99

Cappelluti, N., Brusa, M., Hasinger, G., et al. 2009, A\&A, 497, 635

Chambers, K., \& the Pan-STARRS team. 2006, in The Advanced Maui Optical and Space Surveillance Technologies Conference, 39

Chaudhary, P., Brusa, M., Hasinger, G., Merloni, A., \& Comastri, A. 2010, A\&A, 518, A58

Chaudhary, P., Brusa, M., Hasinger, G., et al. 2012, A\&A, 537, A6

Chiappetti, L., Clerc, N., Pacaud, F., et al. 2012, MNRAS, submitted [arXiv: 1211.4492]

Civano, F., Brusa, M., Comastri, A., et al. 2011, ApJ, 741, 91

Corral, A., Page, M. J., Carrera, F. J., et al. 2008, A\&A, 492, 71

Dalton, G., Trager, S. C., Abrams, D. C., et al. 2012, in SPIE Conf. Ser., 8446

de Jong, R. S., Bellido-Tirado, O., Chiappini, C., et al. 2012, in SPIE Conf. Ser., 8446

Ebrero, J., Carrera, F. J., Page, M. J., et al. 2009, A\&A, 493, 55

Esquej, P., Page, M., Carrera, F. J., et al. 2013, A\&A, 557, A123

Georgakakis, A., Nandra, K., Laird, E. S., Aird, J., \& Trichas, M. 2008, MNRAS, 388, 1205

Gilli, R., Comastri, A., Brunetti, G., \& Setti, G. 1999, New Astron., 4, 45

Hasinger, G., Miyaji, T., \& Schmidt, M. 2005, A\&A, 441, 417

Hasinger, G., Cappelluti, N., Brunner, H., et al. 2007, ApJS, 172, 29

Hiroi, K., Ueda, Y., Akiyama, M., \& Watson, M. G. 2012, ApJ, 758, 49

Hogg, D. W. 1999 [arXiv: astro-ph/9905116]

Kalberla, P. M. W., Burton, W. B., Hartmann, D., et al. 2005, A\&A, 440, 775

Kenter, A., Murray, S. S., Forman, W. R., et al. 2005, ApJS, 161, 9

Kim, M., Wilkes, B. J., Kim, D.-W., et al. 2007, ApJ, 659, 29

Kolodzig, A., Gilfanov, M., Hütsi, G., \& Sunyaev, R. 2013, A\&A, 558, A90

Krumpe, M., Lamer, G., Markowitz, A., \& Corral, A. 2010, ApJ, 725, 2444

La Franca, F., Fiore, F., Comastri, A., et al. 2005, ApJ, 635, 864

Lumb, D. H., Warwick, R. S., Page, M., \& De Luca, A. 2002, A\&A, 389, 93

Merloni, A., Predehl, P., Becker, W., et al. 2012 [arXiv: 1209. 3114]

Miyaji, T., Hasinger, G., \& Schmidt, M. 2000, A\&A, 353, 25

Moretti, A., Campana, S., Lazzati, D., \& Tagliaferri, G. 2003, ApJ, 588, 696

Murray, S. S., Kenter, A., Forman, W. R., et al. 2005, ApJS, 161, 1

Nandra, K., O'Neill, P. M., George, I. M., \& Reeves, J. N. 2007, MNRAS, 382, 194

Perinati, E., Tenzer, C., Santangelo, A., et al. 2012, Exp. Astron., 33, 39

Pierre, M., Pacaud, F., Juin, J. B., et al. 2011, MNRAS, 414, 1732

Predehl, P., Andritschke, R., Böhringer, H., et al. 2010, in SPIE Conf. Ser., 7732

Prokopenko, I. G., \& Gilfanov, M. R. 2009, Astron. Lett., 35, 294

Richards, G. T., Fan, X., Newberg, H. J., et al. 2002, AJ, 123, 2945

Saglia, R. P., Tonry, J. L., Bender, R., et al. 2012, ApJ, 746, 128

Salvato, M., Ilbert, O., Hasinger, G., et al. 2011, ApJ, 742, 61

Shu, X. W., Yaqoob, T., \& Wang, J. X. 2010, ApJS, 187, 581

Silverman, J. D., Green, P. J., Barkhouse, W. A., et al. 2008, ApJ, 679, 118

Snowden, S. L., Egger, R., Freyberg, M. J., et al. 1997, ApJ, 485, 125

Tenzer, C., Warth, G., Kendziorra, E., \& Santangelo, A. 2010, in SPIE Conf. Ser., 7742

Truemper, J. 1993, Science, 260, 1769

Ueda, Y., Akiyama, M., Ohta, K., \& Miyaji, T. 2003, ApJ, 598, 886

Voges, W., Aschenbach, B., Boller, T., et al. 1999, VizieR Online Data Catalog, IX/10

Watson, M. G., Schröder, A. C., Fyfe, D., et al. 2009, A\&A, 493, 339 